Journal of Algebraic Combinatorics 4 (1995), 47-68

(c) 1995 Kluwer Academic Publishers, Boston. Manufactured in The Netherlands.

\title{
Combinatorial $S_{n}$-Modules as Codes*
}

\author{
ROBERT A. LIEBLER \\ Department of Mathematics, Colorado State University Fort Collins, CO 80523 \\ KARL-HEINZ ZIMMERMANN \\ Mathematical Institute, University of Bayreuth, 95440 Bayreuth, Germany
}

\begin{abstract}
Certain $\mathbb{Z} S_{n}$-modules related to the kernels of incidence maps between types in the poset defined by the natural product order on the set of $n$-tuples with entries from $\{1, \ldots, m\}$ are studied as linear codes (when coefficients are extended to an arbitrary field $K$ ). Their dimensions and minimal weights are computed. The Specht modules are extremal among these submodules. The minimum weight codewords of the Specht module are shown to be scalar multiples of polytabloids. A generalization of $t$-design arising from the natural permutation $S_{n}$-modules labelled by partitions with $m$ parts is introduced. A connection with Reed-Muller codes is noted and a characteristic free formulation is presented.
\end{abstract}

Keywords: symmetric group, Specht module, $t$-design, Reed-Muller code

\section{Introduction}

This paper has two purposes, the second of which grew naturally out of the first. Our first purpose is to fill a number of gaps in [11] (cf. Remarks 2.7, 2.13). The second purpose is to point out and extend certain connections between combinatorial $t$-designs, representations of the symmetric group $S_{n}$ labelled by 2-part partitions, and the classical Reed-Muller codes.

We consider the natural permutation representations of the symmetric group to be afforded by $\mathbb{Z}$-free modules over $\mathbb{Z} S_{n}$ and we work with $\mathbb{Z}$-pure submodules over this ring whenever possible. This approach differs from that of many authors who take the coefficient ring to be an arbitrary field $K$. Our approach has many technical advantages and results about $K S_{n}$-modules can be obtained from ours by "extending coefficients" (tensoring with $K$ over $\mathbb{Z}$ ).

The basis of this work is the poset of flags introduced in [11]. Some of its elementary properties as well as a number of alternative formulations are presented in Section 2.1. The incidence maps of this poset are used to introduce $\mathbb{Z}$-forms on certain classical $\mathbb{Q} S_{n^{-}}$ modules in Section 2.2, and our principal object of study $\mathbb{Z} B \kappa_{\lambda}$ is described in Theorem 2.4 and Corollary 2.5. The poset is used to construct large collections of parity checks defined over $\mathbb{Z}$ in Section 2.3. Section 3 presents the most important special cases of the results of Section 4 and may serve as an introduction to this section. The final section presents the combinatorial and coding theoretic connections mentioned above.

*This research was partially supported by NSA grant 904-91-H-0048. 


\section{Background}

\subsection{Notation}

Let $m, n$ be positive integers. A partition $\lambda$ of $n$ having (at most) $m$ parts is a sequence $\left(\lambda_{1}, \ldots, \lambda_{m}\right)$ of non-negative integers such that $\sum_{s=1}^{m} \lambda_{s}=n$. The conjugate partition $\lambda^{\prime}$ to $\lambda$ is defined by $\lambda_{s}^{\prime}=\left|\left\{t \mid s \leq \lambda_{t}\right\}\right|$. The partition $\lambda$ is proper if $\lambda_{s} \geq \lambda_{s+1}$ for all $s$. It turns out that $\lambda=\lambda^{\prime \prime}$ if and only if $\lambda$ is proper. The partition $\lambda$ dominates partition $\nu$ of $n$ $(\lambda \unrhd \nu)$ if $\sum_{s=1}^{t} \lambda_{s} \geq \sum_{s=1}^{t} \nu_{s}$ for all $t$. The domination relation $\unrhd$ defines a partially ordered set (poset) on the partitions of $n$. We say $\lambda$ covers $\nu$ in case the interval $[\nu, \lambda]$ in this poset contains only its endpoints. This means that the diagram of $\lambda$ is obtained from the diagram of $\nu$ by raising one node to the $t$-th from the $(t+1)$-th row (see [8]).

There are three equivalent ways we think about the basis underlying a natural permutation $S_{n}$-module. The most combinatorial mode is flags or tabloids. A flag $\mathbf{F}$ with (at most) $m$ parts is a totally ordered collection $\left(F_{1}, \ldots, F_{m}\right)$ of $m$ subsets of $\{1, \ldots, n\}$ such that $\{1, \ldots, n\}=F_{1} \supseteq \cdots \supseteq F_{m}$. The type of $\mathbf{F}$ is the partition typ $(\mathbf{F})$ of $n$ with $t$-th term $\left|\Delta F_{t}\right|$ where $\Delta F_{t}=F_{t} \backslash F_{t+1}$ for $1 \leq t<m$ and $\Delta F_{m}=F_{m}$. There is a natural (partial) ordering of flags given by $\mathbf{F} \leq \mathbf{G}$ whenever $F_{t} \subseteq G_{t}$ for each $t$. It is easy to see that this partially ordered set is a lattice and

$$
\mathbf{F} \leq \mathbf{G} \text { implies typ }(\mathbf{F}) \unrhd \underline{\operatorname{typ}}(\mathbf{G}) .
$$

If the sets $\Delta F_{t}$ are being emphasized rather than $F_{t}$, the term tabloid rather than flag is used. For instance, the flag $\mathbf{F}=\{\{1, \ldots, 8\},\{2,4,6,8\},\{4,8\}\}$ of type $\left(4,2^{2}\right)$ corresponds to the (3-rowed) tabloid

\begin{tabular}{llll}
\hline 1 & 3 & 5 & 7 \\
\hline 2 & 6 & & \\
\hline 4 & 8 & & \\
\hline
\end{tabular}

The more algebraic mode is to use monomials. A monomial of degree at most $m$ in each variable is an element of $\mathbb{Z}\left[x_{1}, \ldots, x_{n}\right]$ of the form $\prod x_{s}^{i_{s}-1}$ where $1 \leq i_{s} \leq m$. Each such monomial is associated with the unique flag $\mathbf{F}$ where $F_{t}=\left\{s \mid i_{s} \geq t-1\right\}$. Define the type of a monomial by means of this correspondence and observe the relation "is a multiple of" corresponds to $\leq$.

The most succinct mode is sequences. An $n$-tuple $\mathbf{i}=\left(i_{1}, i_{2}, \ldots, i_{n}\right)$ with entries from $\{1, \ldots, m\}$ is associated with the monomial $\prod x_{s}^{i_{s}-1}$ (and so also with a flag). Define the type of a sequence by this correspondence, too. Moreover under this correspondence, "is a multiple of" becomes the natural product order $\leq_{n}$ :

$$
\left(i_{1}, \ldots, i_{n}\right) \leq_{n}\left(j_{1}, \ldots, j_{n}\right) \Leftrightarrow i_{s} \leq j_{s} \text { for all } s \in\{1, \ldots, n\},
$$

which we denote by $\leq$ provided that there is no danger of confusion. Note that infimum $\mathbf{i} \sqcap \mathbf{j}$ and supremum $\mathbf{i} \sqcup \mathbf{j}$ of $\mathbf{i}$ and $\mathbf{j}$ are given explicitly by

$$
\mathbf{i} \sqcap \mathbf{j}:=\left(\min \left\{i_{1}, j_{1}\right\}, \ldots, \min \left\{i_{r}, j_{r}\right\}\right), \mathbf{i} \sqcup \mathbf{j}:=\left(\max \left\{i_{1}, j_{1}\right\}, \ldots, \max \left\{i_{r}, j_{r}\right\}\right)
$$


and denote by $\mathbf{0}$ the least element $(1, \ldots, 1)$ of this lattice. For instance, the above flag $\mathbf{F}$ corresponds to the sequence $(1,2,1,3,1,2,1,3)$ which we abbreviate to 12131213 when there is no danger of confusion.

Let $B=B_{n, m}$ denote this lattice of $n$-tuples on the set $\{1, \ldots, m\}$ (alias flags, tabloids, or monomials). We will use standard lattice theoretic terminology freely, for example a coatom of $[\mathbf{0}, \mathbf{j}]$ is a maximal element among those strictly less than $\mathbf{j}$.

The symmetric group $S_{n}$ acts as an automorphism group on the lattice $(B, \leq)$ by place permutation cf. $[10,3.4 .5]:\left(i_{1}, \ldots, i_{n}\right) \pi=\left(i_{(1)^{\pi}}, \ldots, i_{\left.(n)^{\pi}\right)}\right)$ for all $\pi \in S_{n}$. For each partition $\nu$ of $n$ into $m$ parts, the elements $B^{\nu}$ of $B$ having type $\nu$ form an $S_{n}$-orbit under the above operation. By linear extension of the action of $S_{n}$ on $B$, the free $\mathbb{Z}$ module $\mathbb{Z} B$ with distinguished orthonormal basis $B$ is a right $\mathbb{Z} S_{n}$-module. Denote the associated nondegenerate symmetric bilinear form by $\langle$,$\rangle , so \langle\mathbf{i}, \mathbf{j}\rangle=\delta_{i, j}$ where $\delta$ denotes the Kronecker delta. Now $S_{n}$ acts as a group of orthogonal linear transformations on $\mathbb{Z} B$. The decomposition

$$
\mathbb{Z} B=\oplus \mathbb{Z} B^{\nu} \text { where } \nu \text { ranges over all partitions of } n \text { into } m \text { parts }
$$

is an $\mathbb{Z} S_{n}$-module decomposition. The $\mathbb{Z} S_{n}$-module $\mathbb{Z} B^{\nu}$ is the natural permutation $S_{n}$ module of type $\nu[7,17.4]$.

In spite of equation (1), the Möbius function $\mu^{\prime}$ on the lattice of all partitions of $n$ ordered by dominance and the Möbius function $\mu$ on $(B, \leq)$ are quite different. (For example, $\mu^{\prime}((3,2),(5))=0$ but $\mu(11111,11122)=1$.) Fortunately, we have chosen the easy one to compute.

\section{Lemma 2.1}

(1) $\lambda \unlhd \nu$ if and only if $\mathbf{i} \leq \mathbf{j}$ for some $\mathbf{i} \in B^{\nu}, \mathbf{j} \in B^{\lambda}$.

(2) If $\mathbf{i}, \mathbf{j} \in B^{\nu}$ with $\mathbf{i} \leq \mathbf{j}$ then $\mathbf{i}=\mathbf{j}$.

(3) Let $\mu$ denote the Möbius function on $(B, \leq)$ :

$$
\mu(\mathbf{i}, \mathbf{j})= \begin{cases}(-1)^{t} & \mathbf{i} \text { is infimum of } t \text { distinct coatoms of }[\mathbf{0}, \mathbf{j}] \\ 0 & \text { otherwise. }\end{cases}
$$

Proof: If $\mathbf{i} \leq \mathbf{j}$ then observe that for every positive integer $s$, the number of digits $1, \ldots, s$ occuring in $\mathbf{i}$ is not less than the number of digits $1, \ldots, s$ occuring in $\mathbf{j}$. Hence, $\lambda \unlhd \nu$. Conversely we may take $\mathbf{i}$ and $\mathbf{j}$ as the lexicographically least sequences of type $\nu$ resp. $\lambda$. Thus $\lambda \unlhd \nu$ implies $\mathbf{i} \leq \mathbf{j}$. To prove part 2 observe that two distinct sequences of type $\nu$ belong to the same $S_{n}$-orbit and are thus $\leq$-incomparable (cf. [10, 3.4.4]). Finally, part 3 follows from the fact that the Möbius function is multiplicative on any product order and $\leq$ is composed of $n$ linear orders (cf. [10,2.2]). 


\subsection{Pure submodules and $\mathbb{Z}$-homomorphisms}

As mentioned in the introduction, the $\mathbb{Z} S_{n}$-endomorphisms of $\mathbb{Z} B$ are of central interest to us. Although our use of $\mathbb{Z}$-pure $\mathbb{Z} S_{n}$-submodules is not new (one can find their implicit use in lines -13 to -7 on page 219 of [2]), we wish to emphasize it because we feel that this is the best available setting for the interplay of algebraic and combinatorial methods.

A $\mathbb{Z}$-submodule $P$ of the finitely generated $\mathbb{Z}$-module $M$ is called pure $[3,16.15]$ if the quotient module $M / P$ is torsion free. This is equivalent to the condition that $m \in P$ whenever $0 \neq t \in \mathbb{Z}, m \in M$ and $t m \in P$. In [13] this concept is phrased as " $P$ has index 1 in $M^{\prime \prime}$.

\section{Lemma 2.2 Let $\mathbb{Q}$ be the field of rational numbers and $K$ an arbitrary field.}

(1) The intersection of two pure submodules and the kernel of a $\mathbb{Z}$-homomorphism $\phi$ between finitely generated free $\mathbb{Z}$-modules are pure.

(2) If $P$ is a pure submodule of the free $\mathbb{Z}$-module $M$ then $P$ is also free and its rank equals its dimension when coefficients are extended to $K$.

(3) If two pure submodules determine the same $\mathbb{Q}$-subspace when coefficients are extended to $\mathbb{Q}$, then they coincide.

(4) Suppose $M$ is $\mathbb{Z}$-free and endow it with a nondegenerate bilinear form $\langle$,$\rangle . If N$ is an arbitrary submodule of $M$ then $N^{\perp}$ is pure and $N^{\perp \perp}$ is the smallest pure submodule containing $N$.

Proof: The first statement follows from the definition and the fact that $\mathbb{Z}$ is an integral domain. The second claim appears in $[3,16.16,16.17]$, (and follows directly from the structure theorem for finitely generated modules over a principle ideal domain). The third is immediate from the second and appears in [3, 16.19]. In part four, $N^{\perp}=\cap_{n \in N} \operatorname{ker}\langle *, n\rangle$ is pure by the first claim. The form $\langle$,$\rangle extends to a nondegenerate K$-form, and so $N^{\perp \perp}$ extends to the same $K$-space as the smallest pure submodule containing $N$. Now apply the third part.

The incidence maps $\phi_{\xi}^{\nu}$ and $\psi_{\xi}^{\nu} \in \operatorname{Hom}\left(\mathbb{Z} B^{\nu}, \mathbb{Z} B\right)$ and the projection maps $\pi_{\lambda} \in$ $\operatorname{Hom}\left(\mathbb{Z} B, \mathbb{Z} B^{\lambda}\right)$ are defined by:

$$
\psi_{\xi}^{\nu}(\mathbf{n}):=\sum_{t y p(n \sqcup j)=\xi} \mathbf{j}, \quad \phi_{\xi}^{\nu}(\mathbf{n}):=\sum_{t y p(n \sqcap j)=\xi} \mathbf{j} \text { and } \pi_{\lambda}(\mathbf{i}):=\left\{\begin{array}{l}
\mathbf{i} \text { typ }(\mathbf{i})=\lambda, \\
0 \text { otherwise }
\end{array}\right.
$$

for $\mathbf{n} \in B^{\nu}, \mathbf{i} \in B$. In the case $\nu=\xi$ being of particular importance we obtain by Lemma 2.1:

$$
\psi^{\nu}(\mathbf{n}):=\psi_{\nu}^{\nu}(\mathbf{n})=\sum_{j \leq n} \mathbf{j} \text { and } \phi^{\nu}(\mathbf{n}):=\phi_{\nu}^{\nu}(\mathbf{n})=\sum_{j \geq n} \mathbf{j}
$$




\section{Lemma 2.3}

(1) $\phi_{\xi}^{\nu}, \psi_{\xi}^{\nu}$ and $\pi_{\lambda}$ are $\mathbb{Z} S_{n}$-homomorphisms.

(2) $\left(\operatorname{ker} \pi_{\lambda} \phi_{\xi}^{\nu}\right)^{\perp}=\left(i m \pi_{\nu} \phi_{\xi}^{\lambda}\right)^{\perp \perp}$ and $\left(\operatorname{ker} \pi_{\nu} \psi_{\xi}^{\lambda}\right)^{\perp}=\left(i m \pi_{\lambda} \psi_{\xi}^{\nu}\right)^{\perp \perp}$.

Proof: The first assertion is immediate since $S_{n}$ acts as an automorphism group on the lattice $(B, \leq)$. Assertions 2 and 3 follow from Lemma 2.2 and linear algebra and amount to the observation that $\pi_{\lambda} \phi_{\xi}^{\nu}$ and $\pi_{\nu} \phi_{\xi}^{\lambda}$ resp. $\pi_{\lambda} \psi_{\xi}^{\nu}$ and $\pi_{\nu} \psi_{\xi}^{\lambda}$ are adjoint maps with respect to the form $\langle$,$\rangle .$

We next identify the $\mathbb{Z} S_{n}$-submodule of $\mathbb{Z} B$ that supports representations dominating the representation labelled by $\lambda$. The stabilizer of $1 \in B^{\lambda}$ in $S_{n},\left(S_{n}\right)_{l}:=\left\{\sigma \in S_{n} \mid \mathrm{l} \sigma=1\right\}$ is the Young subgroup associated with $\mathrm{l}$. If $\mathrm{l}^{\prime}$ is a second sequence satisfying $1=\left|\Delta \mathrm{l}_{i} \cap \Delta \mathrm{l}_{j}^{\prime}\right|$ for all nodes $(i, j)$ in the diagram of $\lambda$, then $\mathbf{l}^{\prime}$ has type $\lambda^{\prime}$ and is called a conjugate of 1 [11]. Here $\Delta \mathrm{l}_{t}$ is defined as $\Delta \mathrm{L}_{t}$ where $\mathrm{L}$ is the flag corresponding to $\mathrm{l}$. For $\lambda$ proper, define

$$
\mathbb{Z} B \kappa_{\lambda}:=\left(\mathbb{Z} B \kappa_{l^{\prime}} \mathbb{Z} S_{n}\right)^{\perp \perp} \text { where } \kappa_{l^{\prime}}:=\sum_{\sigma \in\left(S_{n}\right)_{l^{\prime}}} \operatorname{sgn}(\sigma) \sigma \in \mathbb{Z} S_{n} .
$$

In view of the fact that the Specht module $S^{\lambda}$ (as defined below) is generated by $\lambda$ polytabloids [7], the $\perp \perp$ that appears in this definition could be omitted. The careful reader will further note that the assumption that $\lambda$ be proper is really driven by equation (2) below and not essential now.

The (integral) Specht module $S^{\lambda}:=\pi_{\lambda}\left(\mathbb{Z} B \kappa_{\lambda}\right)$ is the central object of study in the module theoretic approach to the symmetric group [7]. In this theory James defines a sequence of modules $S^{\lambda, \nu}$ when $\lambda_{t} \leq \nu_{t}$ for all $t>1$. These (integral) James modules can be expressed in the above language as $\left(\mathrm{n} \kappa_{l^{\prime}} \mathbb{Z} S_{n}\right)^{\perp \perp}$ where $\mathrm{n} \in B^{\nu}$ is obtained from 1 by lowering entries from (only) the first row of 1. An important result of [7] gives an algorithm for finding a submodule series of an arbitrary James module whose terms are Specht modules when coefficients are extended to a field $K$. This result holds also over $\mathbb{Z}$.

For $\mathbf{x}$ of type $\xi$ and $\mathbf{l}^{\prime}$ of type $\lambda^{\prime}$ :

$$
\mathbf{x} \kappa_{l^{\prime}} \neq 0 \text { implies } \lambda^{\prime \prime} \unrhd \xi
$$

because, if $a, b \in\{1, \ldots, n\}$ are in the same row of the tabloid(!) $\mathbf{x}$, i.e., $x_{a}=x_{b}$, then $\mathbf{x}((1)-(a b))=0$. Thus $\mathbf{x} \kappa_{l^{\prime}} \neq 0$ implies that the transposition $(a b)$ is not contained in the Young subgroup associated with $\mathrm{l}^{\prime}$. The Basic Combinatorial Lemma [8] now implies $\lambda^{\prime \prime} \unrhd \xi$.

\section{Theorem 2.4}

(1) $\mathbb{Z} B \kappa_{\lambda}$ is a pure $\mathbb{Z} S_{n}$-submodule of $\mathbb{Z} B$ depending only on $\lambda$ not 1 or $\mathbf{l}^{\prime}$.

(2) If $\lambda$ is proper and $\lambda \unrhd \xi$ and $\phi \in \operatorname{Hom}_{\mathbb{Z} S_{n}}\left(\mathbb{Z} B^{\xi}, \mathbb{Z} B^{\nu}\right), \psi \in \operatorname{Hom}_{\mathbb{Z} S_{n}}\left(\mathbb{Z} B^{\nu}, \mathbb{Z} B^{\xi}\right)$, then

$$
\mathbb{Z} B^{\nu} \kappa_{\lambda} \subseteq(\operatorname{im} \phi)^{\perp} \cap \operatorname{ker} \psi
$$


(3) For $\lambda, \nu$ proper: $\mathbb{Z} B^{\nu} \kappa_{\lambda} \neq 0$ if and only if $\lambda \unrhd \nu$.

(4) For $\lambda$ proper, $\mathbb{Z} B^{\nu} \kappa_{\lambda}=\bigcap_{\lambda \unrhd \xi \llbracket \nu}$ ker $\pi_{\xi} \psi^{\nu}=\bigcap_{\lambda \unrhd \xi} \operatorname{ker} \pi_{\xi} \psi^{\nu}$.

Proof: $\mathbb{Z} B \kappa_{\lambda}$ is pure by Lemma 2.2.4, so part one follows from the fact that $\kappa_{l^{\prime}} \pi=$ $\pi^{-1} \kappa_{l^{\prime}} \pi$ for any $\pi \in S_{n}$. For the second part, note that if $\lambda \notin \xi, \mathbf{j} \in B^{\nu}$ and $\mathbf{l}^{\prime}$ is of type $\lambda^{\prime}$ then the fact that $\langle$,$\rangle is S_{n^{-}}$-invariant, implies $\left\langle\phi(\mathbf{x}), \mathbf{j} \kappa_{l^{\prime}}\right\rangle=\left\langle\phi(\mathbf{x}) \kappa_{l^{\prime}}, \mathbf{j}\right\rangle=\left\langle\phi\left(\mathbf{x} \kappa_{l^{\prime}}\right) \mathbf{j}\right\rangle=0$, by choice of $\phi$ and Equation (2). The second part follows. Part three appears in $[8,1.4 .20]$.

By Lemma 2.2.3, it suffices to establish the final assertion when coefficients are extended to the field $\mathbb{Q}$ of rationals. The second equality follows from the fact that $\pi_{\xi} \psi^{\nu}=0$ unless $\xi \unrhd \nu$. By Lemma 2.3.2 and part two above, $\mathbb{Q} B^{\nu} \kappa_{\lambda} \subseteq \operatorname{ker} \pi_{\xi} \psi^{\nu}$ whenever $\lambda \unrhd \xi$. Suppose $M$ is an irreducible $\mathbb{Q} S_{n}$-submodule of $\bigcap_{\lambda \Downarrow \xi} \operatorname{ker} \pi_{\xi} \psi^{\nu}$. Then $M$ is labelled by a proper partition $\mu \unrhd \nu$ (cf. [10, Section 4.3]) and there is a nontrivial $\mathbb{Q} S_{n}$-homomorphism $\phi: \mathbb{Q} B^{\nu} \rightarrow M \rightarrow S^{\mu}$ that factors through $M$.

If $\lambda \unrhd \mu$, let $\epsilon_{\mu} \in \mathbb{Q} S_{n}$ be the central primitive idempotent of type $\mu$. Then $0 \neq \epsilon_{\mu} \kappa_{l^{\prime}} \in$ $\mathbb{Q} S_{n}$ for $\mathbf{l}^{\prime}$ of type $\lambda^{\prime}$ as above (cf. [8, Section 3.1]). Consequently, there is $\mathbf{l}^{\prime}$ such that $0 \neq M \kappa_{l^{\prime}}$, and so the irreducibility of $M$ implies $M \subseteq \mathbb{Q} B^{\nu} \kappa_{\lambda}$.

Therefore we may suppose $\lambda \unrhd \mu$. Let $A=\left(a_{i j}\right)$ be an upper triangular $m$ by $m$ matrix with having non-negative integer entries with row sums $\left\{\mu_{i}\right\}$ and column sums $\left\{\nu_{j}\right\}$. Define

$$
\begin{aligned}
\psi_{A}:=\pi_{\mu} \psi^{\nu[m]} \cdots \pi_{\nu[k+1]} \psi^{\nu[k]} \cdots \pi_{\nu[2]} \psi^{\nu} ; \\
\text { where } \nu[j]_{i}:= \begin{cases}\sum_{k=1}^{j} a_{i j} & \text { if } i \leq j \\
\nu_{i} & \text { otherwise }\end{cases}
\end{aligned}
$$

are partitions of $n$. Then the image $\psi_{A}(\mathbf{n}), \mathbf{n} \in B^{\nu}$, is the sum of all tabloids $\mathbf{m} \in B^{\mu}$ that have $a_{i j}$ elements from the $j$-th row of $\mathbf{n}$ in the $i$-th row of $\mathbf{m}$. James [7, Theorem 13.13] asserts that these maps (followed by the canonical projection $\pi: \mathbb{Q} B^{\mu} \rightarrow S^{\mu}$ ) form a $\mathbb{Q}$-basis of $\operatorname{Hom}_{\mathbb{Q} S_{n}}\left(\mathbb{Q} B^{\nu}, S^{\mu}\right)$.

Now James' result implies that $\phi \neq 0$ is a $\mathbb{Q}$-linear combination of maps of the form $\pi \psi_{A}$. Consequently $\psi_{A}(M) \neq 0$ for some such matrix $A$. Argue that this is impossible by induction on the number of distinct partitions $\nu[k]$. In the initial case $\mu=\nu, \lambda \unrhd \mu$ does not arise. In the general case, the choice of $M$ in ker $\pi_{\xi} \psi^{\nu}$ for all $\lambda \unrhd \xi$ implies that $\lambda \unrhd \nu[2]$. The result follows by replacing $\nu$ with $\nu[2]$ and applying the induction hypothesis.

Theorem 2.4.2, the first part of proof of Theorem 2.4.4 and Lemma 2.2.2 imply that (cf. $[11,2.6])$

\section{Corollary 2.5}

(1) If $\rho \unrhd \lambda$ are proper then $\mathbb{Z} B^{\nu} \kappa_{\rho} \supseteq \mathbb{Z} B^{\nu} \kappa_{\lambda}$.

(2) The character of $\mathbb{Q} B^{\nu} \kappa_{\lambda}$ is $\sum_{\lambda \Downarrow \zeta \nsubseteq \nu} k_{\zeta, \nu}\{\zeta\}$ where the Kostka number $k_{\zeta, \nu}$ is the multiplicity of the irreducible character $\{\zeta\}$ in $\mathbb{Q} B^{\nu}$.

(3) For $K$ an arbitrary field, the dimension of $K B^{\nu} \kappa_{\lambda}$ is the degree of the above character. 
In order to argue inductively we require a "branching" result. For this, let $\nu(t)$ be the partition obtained from $\nu$ by replacing $\nu_{t}$ by $\nu_{t}-1$ unless $\nu_{t}=0$ when $\nu(t)$ is the partition of 0 . Now define the natural $\mathbb{Z} S_{n-1}$-epimorphism $\pi_{t}: \mathbb{Z} B^{\nu} \rightarrow \mathbb{Z} B^{\nu(t)}$ mapping $\mathbf{j}=\left(j_{1}, \ldots, j_{n}\right) \in B^{\nu}$ to $\left(j_{1}, \ldots, j_{n-1}\right)$ if $j_{n}=t$ and to 0 otherwise.

Corollary 2.6 If $1 \leq t \leq \nu_{1}^{\prime}$ and $\tau=\lambda\left(\lambda_{1}^{\prime}\right)$ then $\pi_{t}\left(\mathbb{Z} B^{\nu} \kappa_{\lambda}\right)=\mathbb{Z} B^{\nu(t)} \kappa_{\tau}$.

Proof: Let $\mathbf{n} \in B^{\nu}, \mathbf{1}^{\prime}$ of type $\lambda^{\prime}$ and suppose $\pi_{t}\left(\mathbf{n} \kappa_{l^{\prime}}\right) \neq 0$. Suppose $n$ is in the $r$-th row of tabloid $\mathrm{l}^{\prime}$ and $\lambda_{r}^{\prime}=\cdots=\lambda_{r+s}^{\prime}>\lambda_{r+s+1}^{\prime}(s \geq 0)$. Now exchange the $r$-th with the $(r+s)$-th row of $\mathrm{l}^{\prime}$, which obviously leaves $\left(S_{n}\right)_{l}$ invariant, and call the resulting element again $\mathbf{l}^{\prime}$. If $\xi^{\prime}$ is the (proper!) partition of $n-1$ obtained from $\lambda^{\prime}$ by replacing $\lambda_{r+s}^{\prime}$ by $\lambda_{r+s}^{\prime}-1$, then $\pi_{t}\left(\mathbf{n} \kappa_{l^{\prime}}\right) \in \mathbb{Z} B^{\nu(t)} \kappa_{\xi}$. Conversely, every generator $\mathbf{m} \kappa_{x^{\prime}}$ of $\mathbb{Z} B^{\nu(t)} \kappa_{\xi}$ can be obtained in this way. Indeed, by appending $t$ at the end of $m$ we obtain a sequence $n$ of type $\nu$. Moreover, appending $n$ to the $(r+s)$-th row of $\xi^{\prime}$ yields an element $l^{\prime}$ of type $\lambda^{\prime}$, and so $\pi_{t}\left(\mathbf{n} \kappa_{l^{\prime}}\right)=\mathbf{m} \kappa_{x^{\prime}}$. Hence,

$$
\pi_{t}\left(\mathbb{Z} B^{\nu} \kappa_{\lambda}\right)=\sum_{\xi} \mathbb{Z} B^{\nu(t)} \kappa_{\xi}
$$

summed over all proper partitions $\xi$ of $n-1$ so that the diagram of $\xi^{\prime}$ is obtained from that of $\lambda^{\prime}$ by deleting one node at the end of its $r$-th row, as $r$ varies. But for each such $\xi, \tau \unrhd \xi$ and $\mathbb{Z} B^{\nu(t)} \kappa_{\tau}$ is a summand of $\pi_{t}\left(\mathbb{Z} B^{\nu} \kappa_{\lambda}\right)$. So by Corollary 2.5 .1 the $\mathbb{Z} S_{n-1}$-modules $\mathbb{Z} B^{\nu(t)} \kappa_{\xi}$ are all contained in $\mathbb{Z} B^{\nu(t)} \kappa_{\tau}$.

Whenever $\mathbf{n} \in B^{\nu}, \mathbf{l}^{\prime}$ of type $\lambda^{\prime}$ and $\mathbf{n} \kappa_{l^{\prime}} \neq 0$, the argument establishing (2) allows us to construct an array whose rows are the rows of $\mathbf{n}$ and whose columns are the rows of $\mathbf{l}^{\prime}$ by placing the number $k$ in the position $(i, j)$ where $\{k\}=\left\{\Delta \mathbf{n}_{i} \cap \Delta \mathbf{l}_{j}^{\prime}\right\}$. In general, the array will have empty positions scattered about, e.g., $\mathbf{n}=11223, \mathbf{l}^{\prime}=32121$. However, if $\mathbf{l} \in B^{\lambda}$ and $\mathbf{l}^{\prime}$ is conjugate to $\mathbf{l}$, one may label node $(i, j)$ of the diagram of $\lambda$ with the element of $\Delta \mathbf{l}_{i} \cap \Delta \mathrm{l}_{j}^{\prime}$ as above and there are no blank positions. The resulting array $T$ is called the $\lambda$-tableau associated with the pair $\left(1,1^{\prime}\right)$ and the element $p_{T}:=1 \kappa_{l^{\prime}}$ is called the $\lambda$-polytabloid based on $T$ [8]. We will show in Theorem 4.4 that the minimum weight words in a Specht module are all multiples of polytabloids.

Remark 2.7 The requirement that $\xi$ takes all values such that $\lambda \unrhd \xi$ in Theorem 2.4 .4 can be weakened, cf. Theorem 5.4.1. The importance of James "kernel intersection theorem" $[7,17.18]$ underscores the value of further improvement of this result.

The (integral) James module $S^{\lambda, \nu}$ is possibly proper submodule of $\mathbb{Z} B^{\nu} \kappa_{\lambda}$. The programs of Eidt [6] provide a starting point of this paper with the following explicit example that $K B^{\nu} \kappa_{\lambda}$ may properly contain the James module $S^{\lambda, \nu}$ when $\nu_{1} \geq \lambda_{2}$ and $\lambda_{t} \leq \nu_{t}$ for all $t>1$. Set $\nu=\left(2^{3}\right), \lambda^{\prime}=\left(2^{2}, 1^{2}\right), \mathbf{n}=112233, \mathbf{l}^{\prime}=121234$, and $\mathbf{k}=121342$. Then $\mathbf{n} \kappa_{k}=112233-211233-132231+231231$ is a generator of $K B^{\nu} \kappa_{\lambda}$, but $\mathbf{n} \kappa_{k}$ is not contained in $S^{\lambda, \nu}=\mathbf{n} \kappa_{l^{\prime}} K S_{n}$. This shows that $[11,2.5,2.6]$ are incorrect for the James modules. 


\subsection{Partial orders and parity checks}

By extending coefficients to a (finite) field $K$, the module $\mathbb{Z} B^{\nu} \kappa_{\lambda}$ and each of its submodules can be viewed as a linear code in $K B^{\nu}$ whose block length is the cardinality of $B^{\nu}$ and where distance is measured relative to the distinguished basis $B^{\nu}$.

In order to establish lower bounds on the minimum distance of these codes, we construct parity checks with disjoint support in all characteristics. For this purpose the characteristic functions of subsets of elements of the lattice introduced at the beginning of this section are ideal. The simplest possible decoding method (cf. Corollary 3.3) appears in case the partitions $\nu$ and $\lambda$ have just two parts and is discussed in Section 3. The first step is to decompose the images of $\phi_{\xi}^{\lambda}$ and $\psi_{\xi}^{\lambda}$ into partial sums.

Let $\mathbf{i}, \mathbf{j} \in B$ and define

$\Phi_{\nu}(\mathbf{i}, \mathbf{j}):=\{\mathbf{n} \mid \mathbf{n} \sqcap \mathbf{j}=\mathbf{i}, \underline{\operatorname{typ}}(\mathbf{n})=\nu\}$ and $\Psi_{\nu}(\mathbf{i}, \mathbf{j}):=\{\mathbf{n} \mid \mathbf{n} \sqcup \mathbf{i}=\mathbf{j}, \underline{\operatorname{typ}}(\mathbf{n})=\nu\}$.

By abuse of notation we also use $\Phi_{\nu}(\mathbf{i}, \mathbf{j})$ and $\Psi_{\nu}(\mathbf{i}, \mathbf{j})$ to denote the sum of all elements in $\Phi_{\nu}(\mathbf{i}, \mathbf{j})$ and $\Psi_{\nu}(\mathbf{i}, \mathbf{j})$, respectively. It will always be clear from the context whether we use $\Phi_{\nu}(\mathbf{i}, \mathbf{j})$ and $\Psi_{\nu}(\mathbf{i}, \mathbf{j})$ as sets or as elements of $\mathbb{Z} B^{\nu}$.

Lemma 2.8 Let $\mathbf{i}, \mathbf{j} \in B$. If $\mathbf{i} \leq \mathbf{j}$ then $\{\mathbf{n} \mid \mathbf{i} \leq \mathbf{n}, \underline{\text { typ }}(\mathrm{n})=\nu\}$ is a disjoint union of sets $\Phi_{\nu}(\mathbf{k}, \mathbf{j})$ taken over all $\mathbf{k} \in[\mathbf{i}, \mathbf{j}]$.

Theorem 2.9 Let $\mathbf{i}, \mathbf{j} \in B$ with $\mathbf{i} \leq \mathbf{j}$.

(1) $\pi_{\nu} \phi^{t y p(i)}(\mathbf{i})=\sum_{k \in[i, j]} \Phi_{\nu}(\mathbf{k}, \mathbf{j})$.

(2) $\Phi_{\nu}(\mathbf{i}, \mathbf{j})=\sum_{k \in[i, j]} \mu(\mathbf{i}, \mathbf{k}) \pi_{\nu} \phi^{t y p(k)}(\mathbf{k})$.

(3) If $\lambda \notin \underline{\operatorname{typ}}(\mathbf{j}), \Phi_{\nu}(\mathbf{i}, \mathbf{j}) \in\left(\mathbb{Z} B^{\nu} \kappa_{\lambda}\right)^{\perp}$.

(4) If $\lambda \not \unrhd \underline{\text { typ}}(\mathbf{i}), i m \pi_{\nu} \phi_{\xi}^{t y p(i)} \subseteq\left(\mathbb{Z} B^{\nu} \kappa_{\lambda}\right)^{\perp}$.

Proof: While the first assertion follows from Lemma 2.8, the second assertion is obtained from the first by Möbius inversion. To prove assertion 3 we conclude from Lemma 2.1.1 and $\lambda \unrhd \operatorname{typ}(\mathbf{j})$ that $\lambda \unrhd \operatorname{typ}(\mathbf{k})$ for all $\mathbf{k} \in[\mathbf{i}, \mathbf{j}]$. Thus in view of Theorem 2.4.2, $\operatorname{im} \pi_{\nu} \phi^{t y p(k)} \subseteq\left(\mathbb{Z} B^{\nu} \kappa_{\lambda}\right)^{\perp}$. Hence the third assertion follows from the second. Finally we conclude from the second assertion that

$\pi_{\nu} \phi_{\xi}^{t y p(i)}(\mathbf{i})=\sum_{x \leq i, t y p(x)=\xi} \Phi_{\nu}(\mathbf{x}, \mathbf{i})=\sum_{x \leq i, t y p(x)=\xi} \sum_{k \in[x, i]} \mu(\mathbf{x}, \mathbf{k}) \pi_{\nu} \phi^{t y p(k)}(\mathbf{k})$.

Moreover, by Lemma 2.1.1, $\lambda \unrhd \underline{\text { typ }}(\mathbf{i})$ implies $\lambda \unrhd \underline{\text { typ }}(\mathbf{k})$ for all $\mathbf{k} \in[\mathbf{x}, \mathbf{i}]$ and $\mathbf{x}$ of type $\xi$. Hence the last assertion follows from Theorem $2 . \overline{4.2}$ and Eq. (3).

Assertions similar to Theorem 2.9.1 and 2.9.2 also hold for $\psi$ and $\Psi$. 
Lemma 2.10 If $\lambda, \xi \unrhd \nu, \lambda \not \beta \xi$ and $\mathbf{j} \in B^{\xi}$ then

$$
P_{\nu, \xi}(\mathbf{j}):=\left\{\Phi_{\nu}(\mathbf{i}, \mathbf{j})-\mu(\mathbf{i}, \mathbf{j}) \pi_{\nu} \phi^{\xi}(\mathbf{j}) \mid \mathbf{i} \in[\mathbf{0}, \mathbf{j}), \mu(\mathbf{i}, \mathbf{j}) \neq 0, \Phi_{\nu}(\mathbf{i}, \mathbf{j}) \neq 0\right\}
$$

forms a set of parity checks for $\mathbb{Z} B^{\nu} \kappa_{\lambda}$ that are, in the usual coding theoretic sense [12, $\mathrm{p}$. 389], orthogonal on $\pi_{\nu} \phi^{\xi}(\mathbf{j})$.

Proof: By Theorem 2.9,

$$
\Phi_{\nu}(\mathbf{i}, \mathbf{j})-\mu(\mathbf{i}, \mathbf{j}) \pi_{\nu} \phi^{\xi}(\mathbf{j})=\sum_{k \in[i, j)} \mu(\mathbf{i}, \mathbf{k}) \pi_{\nu} \phi^{t y p(k)}(\mathbf{k})
$$

But Lemma 2.1.1 shows that $\operatorname{typ}(\mathbf{k}) \triangleright \xi$ and thus $\lambda \unrhd \underline{\operatorname{typ}}(\mathbf{k})$ for each $\mathbf{k} \in[\mathbf{i}, \mathbf{j})$. In view of Theorem 2.4.2, $\Phi_{\nu}(\mathbf{i}, \mathbf{j})-\mu(\overline{\mathbf{i}, \mathbf{j}}) \pi_{\nu} \phi^{\xi}(\mathbf{j})$ is in the orthogonal space of $\mathbb{Z} B^{\nu} \kappa_{\lambda}$. Therefore, Lemma 2.8 implies that $P_{\nu, \xi}(\mathbf{j})$ forms a set of parity checks orthogonal on $\pi_{\nu} \phi^{\xi}(\mathbf{j})$.

The following direct consequence of Lemma 2.10 is remarkably useful.

Lemma 2.11 If $\lambda, \xi \unrhd \nu, \lambda \not \xi$ and $c \in \mathbb{Z} B^{\nu} \kappa_{\lambda}$, then one of the following holds:

(1) $\left\langle\pi_{\nu} \phi^{\xi}(\mathbf{j}), c\right\rangle=0$ for all $\mathbf{j}$ of type $\xi$.

(2) Take $\mathbf{j} \in B^{\xi}$ for which $\left\langle\pi_{\nu} \phi^{\xi}(\mathrm{j}), c\right\rangle \neq 0$ and take $t$ such that $1 \leq t<\xi_{1}^{\prime}$ and define $\mathbf{k}$ as the sequence obtained from $\mathbf{j}$ by replacing all $(t+1)$ 's by $t$ 's. Then the function $\mathbf{j} \sqcap$ - from the sequences $\mathbf{n}$ with $\mathbf{n} \geq \mathbf{k}$ in the support of $c$ to the interval $[\mathbf{k}, \mathbf{j}]$ is onto. Thus, there exist at least $2^{\xi_{t+1}}$ sequences involved in ceach of which has an entry at least as large as that in $\mathbf{j}$ at all positions except where $\mathbf{j}$ has entry $t$ or $t+1$. In case $\xi=\lambda=\nu$ these sequences coincide with $\mathbf{j}$ at all entries different from $t$ and $t+1$.

Proof: Suppose 1. fails and take $\mathbf{j}$ and $t$ as in 2. For $\delta \in\left\{0, \ldots, \xi_{t+1}\right\}$ let $\xi(\delta)$ be the partition obtained from $\xi$ by replacing $\xi_{t}$ with $\xi_{t}+\xi_{t+1}-\delta$ and replacing $\xi_{t+1}$ with $\delta$. The set

$$
I_{t}(\mathbf{j}):=\left\{\mathbf{x} \mid \mathbf{x} \leq \mathbf{j}, \underline{\operatorname{typ}}(\mathbf{x})=\xi(\delta), 0 \leq \delta \leq \xi_{t+1}\right\}
$$

equals the interval $[\mathbf{k}, \mathbf{j}]$. But the latter is a Boolean lattice and so $\mu(\mathbf{i}, \mathbf{j}) \neq 0$ for all $\mathrm{i} \in I_{t}(\mathbf{j})$. Moreover by Lemma $2.10, P_{\nu, \xi}$ belongs to $\left(\mathbb{Z} B^{\nu} \kappa_{\lambda}\right)^{\perp}$. Hence the hypothesis $\left\langle\pi_{\nu} \phi^{\xi}(\mathbf{j}), c\right\rangle \neq 0$ implies that $\Phi_{\nu}(\mathbf{i}, \mathbf{j}) \neq 0$ for all $\mathbf{i} \in[\mathbf{k}, \mathbf{j})$. The result follows.

Corollary 2.12 Let $\lambda \neq(n)$. For each sequence $\mathbf{j}$ of type $\lambda$, the set $P_{\lambda, \lambda}(\mathbf{j})$ equals

$$
\left\{\Phi_{\lambda}(\mathbf{i}, \mathbf{j})-\mu(\mathbf{i}, \mathbf{j}) \mathbf{j} \mid \mathbf{i} \in[\mathbf{0}, \mathbf{j}), \mu(\mathbf{i}, \mathbf{j}) \neq 0\right\}
$$

and is thus a set of $2^{n-\lambda_{1}}-1$ parity checks contained in $\left(S^{\lambda}\right)^{\perp}$ orthogonal on $\mathbf{j}$. 
Hence, for each $\mathrm{j}$ involved in $c \in S^{\lambda}$ and every $t \in\left\{1, \ldots, \lambda_{1}^{\prime}-1\right\}$ there are at least $2^{\lambda_{t+1}}$ sequences involved in $c$ coinciding with $\mathbf{j}$ at all positions with entries $1, \ldots, t-1$, $t+2, \ldots, \lambda_{1}^{\prime}$.

Remark 2.13 Lemma 4.1 provides a kind of converse to Corollary 2.12 .

The claims concerning minimum weight and simple decoding algorithms made in [11, 3.4] assumed that in $P_{\nu, \lambda}(\mathbf{j}), \mu(\mathbf{i}, \mathbf{j}) \neq 0$ for all $\mathbf{i} \in[0, \mathbf{j}]$. As shown in Lemma 2.1.3, this is only correct for 2-part partitions. The presentation of a reasonable decoding algorithm for the general $K B^{\nu} \kappa_{\lambda}$ remains open and seems to be a difficult but worthwhile problem.

\section{2-part partitions}

The above machinery has natural application to combinatorial designs. We pause to make this connection explicit because it motivates the more elaborate arguments to come. The initial cases of these inductive arguments also appears in this section.

Throughout this section $K$ denotes an arbitrary field, $m=2$ and $\nu=(n-k, k)$, $\lambda=(n-\ell, \ell)$ partitions with two parts satisfying $0<\ell \leq k \leq n / 2$. Each sequence $\mathbf{j} \in B^{\nu}$ is identified with the $k$-set of positions where $\mathbf{j}$ has entries 2 . (Equivalently, the tabloid $\mathrm{j}$ is identified with the $k$-set that is its second row.) With this identification, the natural product order $\leq$ of the lattice $(B, \leq)$ is set inclusion.

We begin with an example. Let $\lambda=\nu=(4,3)$. Then $K B^{\lambda}$ can be regarded as the set of $K$-linear combinations of the 3 -sets of $\{1, \ldots, 7\}$. Corollary 2.12 provides seven parity checks for the Specht module $S^{\lambda}$ orthogonal on 567. Namely:

$$
\begin{aligned}
& 567-156-256-356-456,567+125+135+145+235+245+345, \\
& 567-157-257-357-457,567+126+136+146+236+246+346, \\
& 567-167-267-367-467,567+127+137+147+237+247+347, \\
& 567-123-124-134-234 .
\end{aligned}
$$

(In sequence notation the first of these parity checks is $1111222-2111221-1211221-$ 1121221 - 1112221.)

It is well known [8] that $S^{\lambda}$ has dimension 14 . Since $B^{\lambda}$ has cardinality $35, S^{\lambda}$ is a $(35,14)$-code. We will show in Theorem 3.1 that $S^{\lambda}$ has minimum distance 8 . This code is very easy to implement because each message symbol can be correctly decoded by a simple majority vote of such parity checks (cf. Corollary 3.3 ).

A Fano plane is a 2-design with parameters $(7,3,1)$. It is easy to show that any two Fano planes are isomorphic and have full automorphism group of order 168. Therefore a given 7 -set supports $7 ! / 168=30$ different Fano planes. The difference of the characteristic functions of any two Fano planes on the same 7 -set is an element of $S^{\lambda}$. It follows from Corollary 3.2 that any Fano plane is uniquely constructible from any 4 (but not any 3 ) lines.

In the final section we will show how $S^{\lambda}$ considered as binary code is obtainable as a truncation of the classical binary Reed-Muller code $R(3,7)$ with parameters $(128,99,8)$ and investigate more elaborate combinatorial structures that extend the connections illustrated by this example to partitions with more than 2 parts. 
Theorem 3.1 The minimum distance of $K B^{\nu} \kappa_{\lambda}$ is $2^{\ell}$.

Proof: Let $c=\sum c_{i} \mathrm{i}$ be a codeword of $K B^{\nu} \kappa_{\lambda}$ with minimal weight and let $\mathbf{l}^{\prime}$ be of type $\lambda^{\prime}$. We argue by induction on $k-\ell$. If $k=\ell$, then $K B^{\nu} \kappa_{\lambda}$ is the Specht module. Hence by Corollary 2.12 there exist at least $2^{\ell} \ell$-sets involved in $c$. But every generator $p_{T}$ of $S^{\lambda}$ is a $\lambda$-polytabloid and has weight $2^{\ell}$, so $S^{\lambda}$ has minimum distance $2^{\ell}$.

Now let $\ell<k$ and $\xi=(n-\ell-1, \ell+1)$. Since $K B^{\nu} \kappa_{\xi}$ has, by induction, minimum distance $2^{\ell+1}$ and every generator $\mathrm{j} \kappa_{l^{\prime}}$ of $K B^{\nu} \kappa_{\lambda}$ has weight $2^{\ell}$, it follows that $c \notin K B^{\nu} \kappa_{\xi}$.

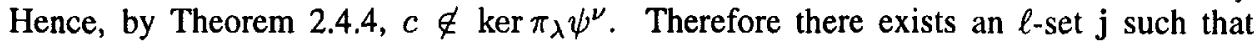
$\left\langle\mathbf{j}, \pi_{\lambda} \psi^{\nu}(c)\right\rangle \neq 0$. But $\left\langle\mathbf{j}, \pi_{\lambda} \psi^{\nu}(c)\right\rangle=\sum_{i \geq j} c_{i}=\left\langle\pi_{\nu} \phi^{\lambda}(\mathbf{j}), c\right\rangle$ and so we conclude from Lemma 2.11 that $K B^{\nu} \kappa_{\lambda}$ has minimum distance $2^{\ell}$.

Corollary 3.2 [4] At-design $S$ is uniquely reconstructible if fewer than $2^{t}$ blocks are lost.

Proof: Suppose $S^{\prime}$ is a second $t$-design sharing all but $2^{t}-1$ blocks with $S$. By assumption, $S^{\prime}$ has the same parameters $t, v, b$ and $k$ as $S$, so the parameter conditions $[12,2.5 .10]$ imply that they have the same parameter $\lambda$ as well. Consequently the difference of their characteristic functions in $\mathbb{Z} B^{(v-k, k)}$ is in the kernel of the incidence map to $t$-sets. Thus $S-S^{\prime} \in \operatorname{ker} \pi_{(v-t, t)} \psi^{(v-k, k)}=\mathbb{Z} B^{(v-k, k)} \kappa_{(v-t-1, t+1)}$, by Theorem 2.4.4, and by construction has weight $\leq 2^{t}-1+2^{t}-1$, contrary to Theorem 3.1 .

Remarkably enough this result is, in some sense, best possible. Indeed, consider the sum $S \in \mathbb{Z} B^{(4,3)}$ of the 7 lines of your favorite Fano plane. Let $\sigma$ be any transposition in the symmetric group $S_{7}$. Then $S^{\sigma}$ is again a Fano plane on the same points but with a slightly different line set and $S-S^{\sigma}$ is of weight 8 in the Specht module $\mathbb{Z} B^{(4,3)} \kappa_{(4,3)}$. Theorem 3.5 implies that this difference is a polytabloid.

\section{Corollary 3.3 The code $S^{\lambda}$ is fully threshold decodable.}

Proof: Let $c$ be a codeword of $S^{\lambda}$ with $\ell$-set $\mathbf{j}$ involved in $c$. For $[\mathbf{0}, \mathbf{j}]$ is a Boolean lattice, $\mu(\mathbf{i}, \mathbf{j}) \neq 0$ for all $\mathbf{i} \in[0, \mathbf{j}]$. Hence, Corollary 2.12 yields a set $P_{\lambda, \lambda}(\mathbf{j})$ of $2^{\ell}-1$ parity checks orthogonal on $\mathbf{j}$. But $S_{n}$ acts as an automorphism group on the product order $\leq$. So $\left[P_{\lambda, \lambda}(\mathbf{j})\right]^{\pi}$ is a set of $2^{\ell}-1$ parity checks orthogonal on $\mathbf{j} \pi$, for each $\pi \in S_{n}$. Since $B^{\lambda}$ is an $S_{n}$-orbit and $S^{\lambda}$ has minimum distance $2^{\ell}$ by Theorem 3.1, the result follows from the definition of threshold decoding (see [12, p. 395] for details).

The last result (and method) goes back at least to Wong [14] in the binary case. Corollary 5.7 gives a multi-step majority decoding algorithm for $K B^{\nu} \kappa_{\lambda}$ that extends the Reed algorithm and the geometric view of the classical Reed-Muller codes.

Lemma 3.4 Suppose $c \in S^{\lambda}$ has minimal weight and $\mathbf{j} \in B^{\lambda}$ is involved in $c$ with coefficient $1_{K}$. Let $\mathcal{C}$ be the set maximal proper subsets of $\mathbf{j}$, i.e. the set of coatoms of $[0, j]$,

(1) For $\mathrm{l} \in \mathcal{C}$ there exists a transposition $\sigma_{l} \in S_{n}$ such that $\mathrm{y}=\mathrm{j} \sigma_{l}$ is the unique $\mathrm{y} \in B^{\lambda}$ which is involved in $c$ and has the property $\mathrm{j} \cap \mathrm{y}=1$. 
(2) For distinct $\mathbf{k}, 1 \in \mathcal{C}$, the transpositions $\sigma_{k}$ and $\sigma_{l}$ commute.

(3) For each $\mathbf{l} \in[\mathbf{0}, \mathbf{j}]$, set $\sigma_{l}:=\prod_{i \in \mathcal{C}, i \geq l} \sigma_{l}$. Then $\mathbf{y}=\mathbf{j} \sigma_{l}$ is the unique $\mathbf{y} \in B^{\lambda}$ that is involved in $c$ and has the property $\mathrm{j} \cap \mathrm{y}=1$.

Proof: By Theorem 3.1, $S^{\lambda}$ has minimum distance $2^{\ell}$. Moreover, Corollary 2.12 and Lemma 2.1.3 show that the set

$$
\left\{\mathbf{j}-\mu(\mathbf{i}, \mathbf{j}) \Phi_{\lambda}(\mathbf{i}, \mathbf{j}) \mid \mathbf{i} \in[\mathbf{0}, \mathbf{j})\right\}
$$

consisting of $2^{\ell}-1$ elements of $\left(S^{\lambda}\right)^{\perp}$ is orthogonal on $\mathbf{j}$. Hence for each $\mathbf{l} \in[0, \mathbf{j})$ there exists exactly one $\ell$-set $\mathbf{j}^{(l)} \in B^{\lambda}$ involved in both $\Phi_{\lambda}(\mathbf{l}, \mathbf{j})$ and $c$. We call this simple observation the uniqueness assertion for $\mathbf{j}$.

Conversely, every $\ell$-set $\mathbf{y}$ involved in $c$ belongs to the set $\Phi_{\lambda}(\mathbf{j} \cap \mathbf{y}, \mathbf{j})$. This establishes the uniqueness assertions in part one and part three.

If $\mathbf{l} \in \mathcal{C}$, then there exists exactly one position $\rho_{l} \in \mathbf{j} \backslash \mathbf{l}$. But $\mathbf{j}^{(l)} \cap \mathbf{j}=\mathbf{l}$ and $\mathbf{j}^{(l)}$ is an $\ell$-set. Hence $\mathbf{j}^{(l)}=1 \cup\left\{\rho_{l}^{\prime}\right\}$ with $\rho_{l}^{\prime} \neq \rho_{l}$. Therefore $\mathbf{j}^{(l)}=\mathbf{j} \sigma_{l}$ where $\sigma_{l}=\left(\rho_{l}^{\prime}, \rho_{l}\right)$, and part one is proved.

Suppose $\mathbf{k}, \mathbf{l} \in \mathcal{C}$ are distinct coatoms of $[\mathbf{0}, \mathbf{j}]$. By the preceding paragraph, there are transpositions $\sigma_{k}=\left(\rho_{k}^{\prime}, \rho_{k}\right)$ and $\sigma_{l}=\left(\rho_{l}^{\prime}, \rho_{l}\right)$ with $\rho_{k}, \rho_{l} \in \mathbf{j}$ and $\rho_{k}^{\prime}, \rho_{l}^{\prime} \notin \mathbf{j}$ so that $\mathbf{j}^{(\mathbf{k})}=\sigma_{\mathbf{k}} \mathbf{j}$ and $\mathbf{j}^{(1)}=\sigma_{\mathbf{l}} \mathbf{j}$. Since $\mathbf{k} \neq \mathrm{l}$ implies $\rho_{k} \neq \rho_{l}$, it suffices to show $\rho_{k}^{\prime} \neq \rho_{l}^{\prime}$. Suppose $\rho_{k}^{\prime}=\rho_{l}^{\prime}$ and put $\mathbf{x}:=\mathbf{k} \cap \mathbf{l}$. But $\mathbf{j} \cap \mathbf{j}^{(x)}=\mathbf{x}$ and $\mathbf{x} \subseteq \mathbf{k}, \mathbf{l} \subseteq \mathbf{j}$ imply $\mathbf{k} \cap \mathbf{j}^{(x)}=\mathbf{x}=\mathbf{l} \cap \mathbf{j}^{(x)}$ and so $\mathbf{j}^{(k)} \cap \mathbf{j}^{(x)}=\left(\mathbf{k} \cup\left\{\rho_{k}^{\prime}\right\}\right) \cap \mathbf{j}^{(x)}=\mathbf{x} \cup\left[\mathbf{j}^{(x)} \cap\left\{\rho_{k}^{\prime}\right\}\right]=\left(\mathbf{l} \cup\left\{\rho_{l}^{\prime}\right\}\right) \cap \mathbf{j}^{(x)}=\mathbf{j}^{(l)} \cap \mathbf{j}^{(x)}$. Therefore $\mathbf{j}^{(k)}$ and $\mathbf{j}^{(l)}$ are both involved in $c$ and in $\Phi_{\lambda}\left(\mathbf{j}^{(k)} \cap \mathbf{j}^{(x)}, \mathbf{j}^{(x)}\right)$, contrary to the uniqueness assertion for $\mathbf{j}^{(x)}$. Part two follows.

Now let $\mathbf{l} \in[\mathbf{0}, \mathbf{j}) \backslash \mathcal{C}$, and fix an atom $\mathbf{a} \in[\mathbf{l}, \mathbf{j}]$, i.e., a minimal element of $(\mathbf{l}, \mathbf{j}]$. Hence $\mathbf{a}=\mathbf{l} \cup\left\{\rho^{\prime}\right\}$ for some $\rho^{\prime}$. Since $\mathbf{j} \cap \mathbf{j}^{(a)}=\mathbf{a}$, there exist coatoms $\mathbf{k} \in[0, \mathbf{j}]$ and $\mathbf{i} \in\left[0, \mathbf{j}^{(a)}\right]$ such that $\mathbf{k} \cap \mathbf{a}=\mathbf{1}=\mathbf{i} \cap \mathbf{a}$. In particular, by part one, $\mathbf{j}^{(k)}=\mathbf{j} \sigma_{k}, \sigma_{k}=\left(\rho_{k}^{\prime}, \rho_{k}\right)$ as above. So $\rho^{\prime} \in \mathbf{j}, \mathbf{k} \cap \mathbf{a}=1$ and $\mathbf{j}=\mathbf{k} \cup\left\{\rho_{k}\right\}$ imply $\rho^{\prime}=\rho_{k}$.

Replacing in part one $\mathbf{j}$ by $\mathbf{j}^{(a)}$ yields a unique $\ell$-set $\mathbf{y}$ involved in $c$ so that $\mathbf{j}^{(a)} \cap \mathbf{y}=\mathbf{i}$ and $\mathbf{y}=\mathbf{j}^{(a)} \sigma$ for some transposition $\sigma$. In particular, $\mathbf{j}^{(a)}=\mathbf{i} \cup\left\{\rho^{\prime}\right\}$ for some $\rho^{\prime}$. But $\mathbf{i} \cap \mathbf{a}=\mathbf{l}$ implies $\rho_{k} \notin \mathbf{i}$ and so it follows from $\mathbf{a} \subseteq \mathbf{j}^{(a)}$ that $\rho^{\prime}=\rho_{k}$. Hence, $\sigma=\left(\rho, \rho_{k}\right)$ and so $\mathbf{y}=\mathbf{i} \cup\{\rho\}$ for some $\rho$.

Claim that $\sigma=\sigma_{k}$. Indeed, suppose $\rho \neq \rho_{k}^{\prime}$. By induction, $\mathbf{j}^{(a)}=\mathbf{j} \sigma_{a}$, where $\sigma_{a}$ is the product of all transpositions $\sigma_{x}$ as $\mathbf{x}$ varies over all coatoms of $[\mathbf{a}, \mathbf{j}]$. But part two shows that $\sigma_{k}$ commutes with each $\sigma_{x}, \mathbf{x}$ coatom of $[\mathbf{a}, \mathbf{j}]$, and so $\rho_{k}^{\prime} \notin \mathbf{j}^{(a)} \supset \mathbf{i}$. Hence, $\mathbf{y} \cap \mathbf{j}^{(k)}=(\mathbf{i} \cup\{\rho\}) \cap\left(\mathbf{k} \cup\left\{\rho_{k}^{\prime}\right\}\right)=(\mathbf{i} \cap \mathbf{k}) \cup(\{\rho\} \cap \mathbf{k})$. Moreover, $\mathbf{y} \cap \mathbf{j}=$ $(\mathbf{i} \cup\{\rho\}) \cap\left(\mathbf{k} \cup\left\{\rho_{k}\right\}\right)=(\mathbf{i} \cap \mathbf{k}) \cup(\{\rho\} \cap \mathbf{k})$ since $\rho_{k} \notin \mathbf{i}$ as shown above. Thus $\mathbf{y} \cap \mathbf{j}=\mathbf{y} \cap \mathbf{j}^{(k)}$ and hence $\mathbf{j}$ and $\mathbf{j}^{(k)}$ belong both to $\Phi_{\lambda}(\mathbf{y} \cap \mathbf{j}, \mathbf{y})$ contradicting the uniqueness assertion for $\mathbf{y}$. Therefore $\sigma=\sigma_{k}$.

Finally claim that $\mathbf{y} \cap \mathbf{j}=\mathbf{l}$ and $\mathbf{y}=\mathbf{j} \sigma_{l}$. Indeed, since $\rho_{k}^{\prime} \notin \mathbf{k}$ and $\mathbf{j}^{(a)}=\mathbf{i} \cup\left\{\rho_{k}\right\}$ as shown above, $\mathbf{y} \cap \mathbf{j}=\left(\mathbf{i} \cup\left\{\rho_{k}^{\prime}\right\}\right) \cap\left(\mathbf{k} \cup\left\{\rho_{k}\right\}\right)=\mathbf{i} \cap \mathbf{k}$. But $\mathbf{l} \subseteq \mathbf{i} \cap \mathbf{k} \subseteq \mathbf{j}^{(a)} \cap \mathbf{j}=\mathbf{a}$ and $\rho_{k} \in \mathbf{a} \backslash(\mathbf{i} \cup \mathbf{k})$ and so $\mathbf{i} \cap \mathbf{k}=1$. Moreover, $\mathbf{k} \cap \mathbf{a}=1$, the definition of $\sigma_{l}$, and the induction hypothesis on $\mathbf{j}^{(a)}$ imply $\mathbf{y}=\mathbf{j}^{(a)} \sigma_{k}=\mathbf{j} \sigma_{l}$ as required. 
Theorem 3.5 The codewords of $S^{\lambda}$ with minimal weight are the $K$-multiples of the $\lambda$ polytabloids.

Proof: Suppose $c$ is codeword of $S^{\lambda}$ with minimal weight and $\mathbf{j}$ is involved in $c$ with coefficient $1_{K}$. By Lemma 3.4.3, $\mathrm{j} \sigma_{l}$ is involved in $c$ for each $l \in[0, \mathbf{j}]$. Since $c$ has minimal weight, it follows that $c=\sum_{l \in[0, j]} c_{l} \mathrm{j} \sigma_{l}\left(c_{l} \in K\right)$ and, by the choice of $c, c_{j}=1$. If $\mathbf{l} \in[0, j)$, then Corollary 2.12 and the uniqueness assertion for $j$ stated in the proof of Lemma 3.4 imply,

$$
0=\left\langle\Phi_{\lambda}(\mathbf{l}, \mathbf{j})-\mu(\mathbf{l}, \mathbf{j}) \mathbf{j}, c\right\rangle=\left\langle\mathbf{j} \sigma_{l}-\mu(\mathbf{l}, \mathbf{j}) \mathbf{j}, c_{l} \mathbf{j} \sigma_{l}+\mathbf{j}\right\rangle=c_{l}-\mu(\mathbf{l}, \mathbf{j}),
$$

so $c_{l}=\mu(\mathbf{l}, \mathbf{j})=(-1)^{t}$, where $t$ is the number of coatoms in $[\mathbf{l}, \mathbf{j}]$ by Lemma 2.1.3. Hence $c_{l}=\operatorname{sgn}\left(\sigma_{l}\right)$ by definition of $\sigma_{l}$.

We now construct a $\lambda$-tableau $T$ whose first and second row respectively is formed by $\{1, \ldots, n\} \backslash \mathbf{j}$ and $\mathbf{j}$. For each coatom $\mathbf{l}$ of $[\mathbf{0}, \mathbf{j}]$ there is by Lemma 3.4 a corresponding transposition $\sigma_{l}=\left(\rho_{l}, \rho_{l}^{\prime}\right)$ with $\rho_{l} \in \mathbf{j}$ and $\rho_{l}^{\prime} \notin \mathbf{j}$. But the transpositions corresponding to distinct coatoms of $[0, \mathrm{j}]$ commute and so we may arrange the entries of $T$ so that $\rho_{l}^{\prime}$ appears in a column above $\rho_{l}$. Hence the column group of $T$ is generated by the set $\left\{\sigma_{l} \mid 1 \in \mathcal{C}\right\}$. Thus by the first paragraph, $c=p_{T}$ is a polytabloid.

Theorem 3.5 does not extend to $\mathbb{Z} B^{\nu} \kappa_{\lambda}$. Indeed, let $\lambda=(4,2)$ and $\nu=\left(3^{2}\right)$. Then $126-125-346+345=(126-136-126+135)+(136-345-135+345)$ is the sum of two generators and has minimal weight by Theorem 3.1, but is not a generator.

\section{Minimum weight words and polytabloids}

We now consider the modules $K B^{\nu} \kappa_{\lambda}, K$ an arbitrary field, as linear codes in $K B^{\nu}$. It is conceivable that the minimum weight of $K B^{\nu} \kappa_{\lambda}$ be strictly less than the minimum weight of $\mathbb{Z} B^{\nu} \kappa_{\lambda}$ because the latter might have a word with many (but not all!) coordinates divisible by the characteristic of $K$. The fact that this is not the case, may be the first clue that these codes are not "good codes".

The first part of this section determines the minimum weight words in the Specht modules, Theorem 4.4. Lemma 4.1 shows how to use Lemma 3.4 to associate a tableau to $c \in S^{\lambda}$ whenever equality holds for each $t$ in Corollary 2.12 . We then describe an algorithm that constructs a set of $(t+1)^{\lambda_{t+1}}$ sequences involved in $c$ that agree in all positions except at their entries $t+1$. When applied to minimal weight elements of $S^{\lambda}$ equality is forced to hold for each $t$ in Corollary 2.12.

The last part of this section uses Theorem 4.4 as the basis of an inductive argument that shows the minimal weight of $K B^{\nu} \kappa_{\lambda}$ depends only on $\lambda$. In this argument we make essential use of the fact that we have resisted the temptation to force $\nu$ to be proper in the forgoing discussion.

Lemma 4.1 Let $\lambda \neq(n)$ and let $c$ be a non-zero codeword of $S^{\lambda}$. Suppose for every $\mathbf{j}$ involved in $c$ and for every $t, 1 \leq t<\lambda_{1}^{\prime}$, there are exactly $2^{\lambda_{t+1}}$ sequences involved in $c$ which coincide with $\mathbf{j}$ at all positions with entries different from $t$ and $t+1$. Then there 
exists a $\lambda$-tableau $T, k \in K \backslash\{0\}$, and $c^{\prime} \in S^{\lambda}$ so that $c=k p_{T}+c^{\prime}$ and all sequences involved in $p_{T}$ are also involved in $c$.

Proof: Suppose $\mathbf{j}$ is involved in $c$ with coefficient 1 . For every $t, 1 \leq t<\lambda_{1}^{\prime}$, we may invoke Lemma 3.4 with $I_{t}(\mathbf{j})$ (cf. proof of Lemma 2.11) instead of $[0, \mathbf{j}]$ to construct a partial sum $c^{(t)}$ of $2^{\lambda_{t+1}}$ sequences involved in $c$ which turns out to be a polytabloid for some $\left(\lambda_{t}, \lambda_{t+1}\right)$-tableau $T_{t}$ when puncturing, i.e., deleting all positions with entries $1, \ldots, t-1, t+2, \ldots, \lambda_{1}^{\prime}$. Clearly we may choose $T_{t}$ so that $x$ is in the first row of $T_{t}$ if and only if $\mathrm{j}$ has entry $t$ at position $x$.

Construct a $\lambda$-tableau $T$ from the given tableaux $T_{t}$ as follows: Let the next to the last and the last row of $T$ be the first resp. second row of $T_{\lambda_{1}^{\prime}-1}$. The choice of the tableaux $T_{t}$ assures that the first row of $T_{\lambda_{1}^{\prime}-1}$ and the second row of $T_{\lambda_{1}^{\prime}-2}$ coincide up to some row permutation. Now permute the columns of $T_{\lambda_{1}^{\prime}-2}$ so that the first row of $T_{\lambda_{1}^{\prime}-1}$ coincides entry by entry with the second row of the permuted $T_{\lambda_{1}^{\prime}-2}$ and take the first row of the permuted $T_{\lambda_{1}^{\prime}-2}$ as the third to the last row of $T$. By proceeding in this way, obtain a $\lambda$-tableau $T$. Since all of this started from a fixed $\mathbf{j}, T$ is well-defined.

Suppose $E$ is the set of all permutations in the column group of $T$ fixing all numbers of $T$ up to those in rows $t$ and $t+1$ as $t$ varies. Hence, by construction,

$$
c^{(t)}=\sum_{\sigma} \operatorname{sgn}(\sigma) \mathbf{j} \sigma
$$

summed over all $\sigma \in E$ fixing all entries of $T$ outside of rows $t$ and $t+1$.

Now let $\sigma_{0} \in E, \sigma_{0} \neq 1$, fixing all numbers of $T$ up to those in rows $s$ and $s+1$. Since $\mathbf{j} \sigma_{0}$ is involved in $c$ we may similarly construct for every $t, 1 \leq t<\lambda_{1}^{\prime}$, a partial sum $c_{0}^{(t)}$ of sequences involved in $c$ which is a polytabloid for some $\left(\lambda_{t}, \lambda_{t+1}\right)$-tableau when deleting all positions with entries $1, \ldots, t-1, t+2, \ldots, \lambda_{1}^{\prime}$. But $\mathrm{j} \sigma_{0}$ is involved in $c$ with coefficient $\operatorname{sgn}\left(\sigma_{0}\right)$ and so we obtain

$$
c_{0}^{(t)}=\operatorname{sgn}\left(\sigma_{0}\right) c^{(t)} \sigma_{0} .
$$

Therefore, $c_{0}^{(s)}=c^{(s)}$ and $c_{0}^{(t)}$ and $c^{(t)}$ have disjoint support when $s \neq t$. Conclude from (4) and (5) that for every $\sigma \in E, \mathrm{j} \sigma \sigma_{0}$ is involved in $c$ with coefficient $\operatorname{sgn}\left(\sigma_{0} \sigma\right)$.

Since $E$ generates the column group of $T$ we may apply the above argument to show by induction on the number of generators that all sequences involved in $p_{T}$ are involved in $c$, too. Now the proof is complete.

It is now time to present the algorithm that is used to "fatten up" $c \in S^{\lambda}$ so that Lemma 4.1 applies. The algorithm makes heavy use of Corollary 2.12 . We first illustrate the idea by an example:

Suppose $\mathbf{j}=11111222233344$ is involved in $c \in S^{(5,4,3,2)}$. From $\mathbf{j}^{(0)}:=\mathbf{j}$ we may construct in turn sequences $\mathbf{j}^{(l+1)}$ involved in $c$ whose entries $t+1$ are located at positions at which $\mathbf{j}$ has entries $t-\iota(\iota<t \leq 3)$. The overwritten $(\iota+1)$ 's will then fill the vacant spots, e.g., $\mathbf{j}^{(1)}=22221333144111, \mathbf{j}^{(2)}=33321442122111$, and $\mathbf{j}^{(3)}=44321332122111$. We may modify this construction to leave one or both entries 4 at their positions. For instance, we may obtain from $\mathbf{j}$ in turn $\mathbf{I}^{(1)}=22221333141114$ by fixing the second 4 at the last 
position, $\mathrm{l}^{(2)}=33321422121114$, and $\mathrm{l}^{(3)}=34321312121114$. For our purposes it is irrelevant which positions with entries $t$ are replaced by $(t+1)$ 's. When the construction (and notation) appearing the proof of Lemma 4.2 is applied to this example one obtains the sequences: $(t=3) \mathbf{j}^{(1)}=\mathbf{k}^{(3,3)}, \mathbf{j}^{(2)}=\mathbf{k}^{(2,2)}, \mathbf{j}^{(3)}=\mathbf{k}^{(1,1)}, \mathbf{l}^{(1)}=\mathbf{k}^{(3,4)}, \mathbf{l}^{(2)}=\mathbf{k}^{(2,4)}$, and $\mathrm{l}^{(3)}=\mathbf{k}^{(1,4)}$.

Lemma 4.2 Let $\mathbf{j}$ be a sequence involved in $c \in S^{\lambda}, \lambda \neq(n)$. Let $1 \leq t<\lambda_{1}^{\prime}$. For each $\underline{z} \in\{1, \ldots, t+1\}^{\lambda_{t+1}}$ there exists a sequence $\mathbf{k}^{\underline{z}}$ involved in $c$ with the following properties:

(1) $\mathbf{k}$ coincides with $\mathbf{j}$ at all positions with entries $>t+1$.

(2) For distinct $\underline{z}, \underline{z}^{\prime} \in\{1, \ldots, t+1\}^{\lambda_{t+1}}$ the sequences $\mathbf{k}^{\underline{z}}$ and $\mathbf{k}^{\underline{z}^{\prime}}$ can be distinguished by only looking at positions with entries $t+1$.

Proof: Adopt the notation of the proof of Lemma 2.11. Let $\underline{z} \in\{1, \ldots, t+1\}^{\lambda_{t+1}}$ and suppose $y_{1}>\cdots>y_{q}$ are the distinct entries of $\underline{z}$.

We recursively construct a sequence $\mathbf{k}^{(0)}:=\mathbf{j}, \mathbf{k}^{(1)}, \ldots, \mathbf{k}^{\left(t+1-y_{q}\right)}=: \mathbf{k}^{z}$ of elements involved in $c$ each of which has the following properties:

$A_{1}^{L}: \mathbf{k}^{(\iota)}$ coincides with $\mathbf{j}$ at all positions with entries $>t+1$.

$A_{2}^{\iota}: \mathbf{k}^{(\iota)}$ has all entries $t^{\prime}$ at positions at which $\mathbf{j}$ has entries $t^{\prime}-\iota\left(\iota<t^{\prime} \leq t\right)$.

$A_{3}^{\iota}$ : The set $X_{\iota}:=\left\{x_{1}<\cdots<x_{m_{\iota}}\right\}$ of all positions at which $\mathbf{k}^{(\iota)}$ has entries $t+1$ and $\mathrm{j}$ has entries $t-\iota+1$ is nonempty and the sequence obtained from $\underline{z}$ by deleting all components greater than $t+1-\iota$ is an $\left|X_{t}\right|$-tuple.

When $\underline{z}$ contains the entry $y_{s}=t+1-\iota$, as in condition $A_{3}^{\iota}$, let $\underline{z}^{(s)}$ denote the tuple obtained from $\underline{z}$ by deleting all components $>y_{s}$ and define $Y_{\iota} \subset X_{\iota}$ as follows:

$$
x_{i} \in Y_{\iota}: \Leftrightarrow y_{s} \text { occurs at position } i \text { in } \underline{z}^{(s)} \text {. }
$$

In view of $A_{3}^{\iota}, Y_{\iota}$ is well-defined. Moreover $Y_{\iota}$ is a proper subset of $X_{\iota}$ since $\iota<t+1-y_{q}$. (If $\underline{z}$ contains no entry $t+1-\iota$, put $Y_{\iota}:=\emptyset$.)

Suppose $\mathbf{k}^{(\iota)}$ has already been constructed $\left(0 \leq \iota \leq t-y_{q}\right)$. The construction of $\mathbf{k}^{(\iota+1)}$ from $\mathbf{k}^{(\iota)}$ and $\underline{z}$ (resp. $X_{\iota}$ and $Y_{\iota}$ ) is itself accomplished by constructing a sequence $\mathbf{x}^{(t+1)}, \ldots, \mathbf{x}^{(t+1)}$ terminating in $\mathbf{k}^{(\imath+1)}$ each of whose terms is involved in $c$ :

(i): Start with $\mathbf{x}^{(t+1)}=\mathbf{k}^{(\iota)}$ and choose $\mathbf{i}^{(t)} \in I_{t}\left(\mathbf{k}^{(\iota)}\right)$ of type $\xi\left(\lambda_{t+1}-\left|X_{\iota} \backslash Y_{\iota}\right|\right)$ differing from $\mathbf{k}^{(\iota)}$ at the positions in $X_{\iota} \backslash Y_{\iota}$. Construct a sequence $\mathbf{x}^{(t)}$ from $\mathbf{k}^{(\iota)}$ by replacing all entries at positions in $X_{\iota} \backslash Y_{\iota}$ by $t$ 's and $\left|X_{\iota} \backslash Y_{t}\right|$ entries $t$ by $(t+1)$ 's so that $\mathbf{x}^{(t)}$ coincides with $\mathbf{k}^{(\iota)}$ at all positions in $Y_{\iota}$ where both contain entries $t+1$. Then $\mathbf{x}^{(t)}$ is involved in both $c$ and $\Phi_{\lambda}\left(\mathbf{i}^{(t)}, \mathbf{k}^{(\iota)}\right)$.

(ii): Suppose $\mathbf{x}^{\left(t^{\prime}+1\right)}$ already has been constructed $\left(\iota<t^{\prime}<t\right)$. Choose the least element $\mathbf{i}^{\left(t^{\prime}\right)}$ of $I_{t^{\prime}}\left(\mathbf{x}^{\left(t^{\prime}+1\right)}\right)$. Construct a sequence $\mathbf{x}^{\left(t^{\prime}\right)}$ from $\mathbf{x}^{\left(t^{\prime}+1\right)}$ by replacing each $t^{\prime}+1$ by $t^{\prime}$ and $\lambda_{t^{\prime}+1}$ entries $t^{\prime}$ by $\left(t^{\prime}+1\right)$ 's. Then $\mathbf{x}^{\left(t^{\prime}\right)}$ involved in both $c$ and $\Phi_{\lambda}\left(\mathbf{i}^{\left(t^{\prime}\right)}, \mathbf{x}^{\left(t^{\prime}+1\right)}\right)$. 
We must verify that $\mathbf{k}^{(\iota+1)}:=\mathbf{x}^{(\iota+1)}$ fulfills the assertions $A_{i}^{\iota+1}$, for $i=1,2,3$. The construction ensures that $\mathbf{x}^{(t)}, \ldots, \mathbf{x}^{(\iota+1)}$ coincide at all positions with entries $\geq t+1$. Hence, by $A_{1}^{\iota}$ and part (i), $\mathbf{k}^{(\iota+1)}$ satisfies $A_{1}^{\iota+1}$.

In view of $A_{2}^{\iota}, \mathbf{k}^{(\iota)}$ contains all its entries $t$ at positions at which $\mathbf{j}$ has entries $t-\iota$. Thus by part (i), $X_{\iota+1}=X_{\iota} \backslash Y_{\iota} \neq \emptyset$ is the set of all positions at which $\mathbf{k}^{(\iota+1)}$ has entries $t+1$ and $\mathbf{j}$ contains $(t-\iota)$ 's. Thus in view of the definition of $Y_{\iota}, \underline{z}^{(s+1)}$ is a $\left|X_{\iota+1}\right|$-tuple. Hence $A_{3}^{\iota+1}$ follows.

Finally, $\mathbf{k}^{(\iota)}$ and $\mathbf{x}^{\left(t^{\prime}+1\right)}$ coincide at all positions with entries $<t^{\prime}+1$, while $\mathbf{k}^{(\iota+1)}$ and $\mathbf{x}^{\left(t^{\prime}\right)}$ coincide at all positions with entries $\geq t^{\prime}+1\left(\iota<t^{\prime}<t\right)$. Hence part (ii) shows that $\mathbf{k}^{(\iota+1)}$ has all entries $t^{\prime}+1$ at positions at which $\mathbf{k}^{(\iota)}$ has entry $t^{\prime}\left(\iota<t^{\prime}<t\right)$. So $A_{2}^{\iota}$ implies $A_{2}^{\iota+1}$.

This completes the construction of the sequence $\mathbf{k}^{(0)}, \mathbf{k}^{(1)}, \ldots, \mathbf{k}^{\left(t+1-y_{q}\right)}$. All that remains is to verify that $\mathbf{k}^{\underline{z}}:=\mathbf{k}^{\left(t+1-y_{q}\right)}$ satisfies assertions 1 and 2 . The verification of $A_{3}^{\iota+1}$ shows that $\mathbf{k} \underline{z}$ satisfies assertion 1 .

For distinct tuples $\underline{z}$ and $\underline{z}^{\prime}$ having least entries $y_{q}$ and $y_{q^{\prime}}^{\prime}$ respectively, the associated families $\left(Y_{0}, \ldots, Y_{t-y_{q}}\right)$ resp. $\left(Y_{0}^{\prime}, \ldots, Y_{t-y_{q^{\prime}}^{\prime}}^{\prime}\right)$ of sets of positions specified in the above recursive construction are distinct $\left(Y_{\iota} \neq Y_{\iota}^{\prime}\right.$ for some $\iota$ ).

But the above construction shows that $Y_{t+1-y_{s}}$ is the set of positions at which $\mathbf{k}^{\underline{z}}$ has entries $t+1$ and $\mathbf{j}$ has entries $y_{s}$ for $1 \leq s<q$. (Moreover, the remaining $(t+1)$ 's of $\mathbf{k} z$ are located at positions at which $\mathrm{j}$ has entries $y_{q}$.) Hence assertion 2 follows.

Lemma 4.3 Let $c$ be a codeword of $S^{\lambda}$ such that $\mathrm{j}$ is involved in $c$ and let $1 \leq t<\lambda_{1}^{\prime}$. Then there exist at least $\prod_{s=1}^{t+1} s^{\lambda_{s}}$ sequences involved in c coinciding with $\mathbf{j}$ at all positions with entries $>t+1$.

Proof: We argue by induction. Corollary 2.12 proves the assertion for $t=1$. Suppose the lemma is proved for $t<\lambda_{1}^{\prime}$. Then applying the induction hypothesis to each of the $(t+1)^{\lambda_{t+1}}$ sequences $\mathbf{k}^{\underline{z}}$ constructed in Lemma 4.2 yields the result.

Theorem 4.4 The codewords of Specht module $S^{\lambda}$ with minimal weight are the $K$ multiples of the $\lambda$-polytabloids.

Proof: It suffices to show that every codeword of $S^{\lambda}$ with minimal weight fulfills the hypothesis of Lemma 4.1. Indeed, this is clear for $\lambda_{1}^{\prime}=2$, by Theorem 3.1. Now suppose $\lambda_{1}^{\prime}>2$ and $c$ is a codeword of minimal weight. Then it follows from the induction argument used in the proof of Lemma 4.3 that for every $\mathrm{j}$ involved in $c$ and for every $t, 1 \leq t<\lambda_{1}^{\prime}$, there are exactly $\prod_{s=1}^{t+1} s^{\lambda_{s}}$ sequences involved in $c$ which coincide with $\mathbf{j}$ at all positions with entries $>t+1$. Hence Lemma 4.3 can be used to construct all sequences involved in $c$.

So if $\mathbf{j}$ is involved in $c$, then by Lemma 4.2 , we obtain for each $\underline{z} \in\{1, \ldots, t+1\}^{\lambda_{t+1}}$ a sequence $\mathbf{k}^{\underline{z}}$ involved in $c$ coinciding with $\mathbf{j}$ at all positions with entries $>t+1$, and in turn a set of exactly $\prod_{s=1}^{t} s^{\lambda_{x}}$ sequences involved in $c$ coinciding with $\mathbf{k}^{\underline{z}}$ at all positions 
with entries $>t$. Moreover the sets of sequences $\prod_{s=1}^{t} s^{\lambda_{s}}$ involved in $c$ constructed from distinct tuples $\underline{z}$ and $\underline{z}^{\prime}$ are disjoint. Hence each sequence involved in $c$ coinciding with $\mathbf{j}$ at all positions with entries different from $t$ and $t+1$ must lie in a set of $\prod_{s=1}^{t} s^{\lambda_{s}}$ sequences constructed from $\mathrm{k} \underline{\underline{z}}$ where $\underline{z} \in\{t, t+1\}^{\lambda_{t+1}}$. But by construction, in every set of $\prod_{s=1}^{t} s^{\lambda_{s}}$ sequences constructed from $\mathbf{k} \underline{z}\left(\underline{z} \in\{t, t+1\}^{\lambda_{t+1}}\right)$ there is at most one member coinciding with $\mathrm{j}$ at all positions with entries different from $t$ and $t+1$. Hence by Corollary 2.12 there are exactly $2^{\lambda_{t+1}}$ sequences with the required properties. This completes the proof.

We now turn to $K B^{\nu} \kappa_{\lambda}$. As mentioned earlier, the argument is inductive on $n$ and involves improper $\nu$. The epimorphisms $\pi_{i}$ introduced before 2.6 also come into play.

Lemma 4.5 Let $0 \neq c \in K B^{\nu} \kappa_{\lambda}$. Then $\pi_{i}(c) \neq 0$ for $\lambda_{1}^{\prime}$ distinct $i \in\{1, \ldots, m\}$.

Proof: Reorder the parts of $\nu$ so that $\pi_{i}(c) \neq 0$ for all $1 \leq i \leq t$. Argue by induction on $|[\nu, \lambda]|$ that $t \geq \lambda_{1}^{\prime}$. In case $\lambda=\nu$ or $c \in S^{\nu}$, Theorem 4.4 implies the result.

Now assume $c \notin S^{\nu}$. Then an important result of James [7, 17.13] implies that there are integers $s, v$ and a partition $\mu$ obtained from $\nu$ by adding $v$ to some $\nu_{s}$ and subtracting $v$ from $\nu_{s+1}$ such that $\pi_{\mu} \psi^{\nu}(c) \neq 0$. Theorem 2.4.2 implies that $\mu \in(\nu, \lambda \mid$. By construction of $\mu$ and the initial reordering, the only positions where some $\mathbf{n} \in B^{\nu}$ differs from some $\mathbf{m} \in B^{\mu}$ in the support of $\pi_{\mu} \psi^{\nu}(\mathbf{n})$ is in positions where $s+1$ appears in $\mathbf{n}$ and the entries of $\mathbf{m}$ in these positions are either $s$ or $s+1$. Thus by our hypothesis on $c, n$ is such a position only if $s<t$. It follows that $\pi_{i} \pi_{\mu} \psi^{\nu}(c) \neq 0$ can only occur if $i \leq t$. The induction hypothesis implies $t \geq \lambda_{1}^{\prime}$ as desired.

\section{Theorem 4.6 The minimum weight of $K B^{\nu} \kappa_{\lambda}$ is $\prod_{s \geq 1} s^{\lambda_{a}}$.}

Proof: Argue by induction on $n$. The initial case is trivial. Suppose the theorem holds for $S_{n-1}$ and $0 \neq c \in K B^{\nu} \kappa_{\lambda}$. Lemma 4.5 implies that $\pi_{t}(c) \neq 0$, for at least $\lambda_{1}^{\prime}$ distinct values of $t$. The induction hypothesis and Corollary 2.6, imply that $\pi_{t}(c)$ has weight at least equal to $\left(\prod_{s} \lambda_{s}^{\prime} !\right) / \lambda_{1}^{\prime}$ for each such $t$. The theorem now follows from the fact that the preimages of $\pi_{t}(c)$ have disjoint support (as partial sums of $c$ ) and $\prod_{s} \lambda_{s}^{\prime} !=\prod_{s} s^{\lambda_{x}}$.

\section{Applications}

\subsection{Designs}

Recall that a combinatorial $t$-design is a collection of $k$-sets of an $n$-set with the property that each $t$-set $(t<k \leq n / 2)$ is contained in a constant number $\lambda$ of elements of the collection. It is well known $[12,21.9]$ that the characteristic function of the set of blocks of a $t$-design has trival projection into the first through $t$-th representation (eigen) spaces of the Johnson Scheme $J(n, k)$.

In contrast to the Johnson scheme, the incidence maps associated with partitions having more than two parts arise in $\operatorname{Hom}_{\mathbb{Z} s_{n}}(\mathbb{Z} B, \mathbb{Z} B)$ as presented in Section 2. The analogous 
representations of $\operatorname{Hom}_{\mathbb{Z} S_{n}}\left(\mathbb{Z} B^{\nu}, \mathbb{Z} B^{\nu}\right)$ are however not totally ordered in a natural way as in $J(n, k)$. Instead the total order for $J(n, k)$ extends to the domination partial order.

In an effort to extend the combinatorial interpretation illustrated at the beginning of Section 3, we are led to identify an element of $\mathbb{Z} B^{\nu}$ having all coordinate values in $\{0,1\}$ with the set of flags in its support and to make:

Definition 5.1 A $\tau$-design of type $\nu$ is a subset $S \subset B^{\nu}$ such that $\pi_{\tau} \psi^{\nu}(S)=\lambda_{\tau} B^{\tau}$ for some integer $\lambda_{\tau}>0$. In order to avoid degeneracies, we further require that $\nu_{1} \geq n / 2$ and $\tau \unrhd \nu$.

In order to maintain notation consistent with the 2-part case, we may omit the first part of $\tau$ and $\nu$ writing simply " $\tau_{2}, \ldots, \tau_{m}$-design of type $\nu_{2}, \ldots, \nu_{m}$ " rather than the more formal $\left(\tau_{1}, \tau_{2}, \ldots, \tau_{m}\right)$-design of type $\left(\nu_{1}, \nu_{2}, \ldots, \nu_{m}\right)$. Higher type designs are perhaps best viewed in the "flag mode" of Section 2 . Thus, for example, a 1,1-design $S$ of type $a, b$, consists of a set of blocks, each of which is an ordered pair $(X, Y)$ of sets, such that $Y \subseteq X \subseteq\{1, \ldots, n\},|X|=a+b,|Y|=b$ and there is an integer $\lambda$ such that for any ordered pair of distinct points $(x, y)$ the number of blocks $(X, Y)$ with $x \in X, y \in Y$ is always $\lambda$, independent of the choice of $(x, y)$.

Some $\tau$-designs of higher type that are not just "warmed over" $t$-designs come from finite geometry. For example, the incident line, plane pairs in the projective geometry $\operatorname{PG}(n, q)$ form a $1^{2}$-design of type $q^{2}, q+1$. Also, a resolvable 2-design with $t+1$ blocks of size $k$ per parallel class is equivalent to a $0^{t-1}, 2$-design of type $k^{t}$.

It would be of desirable to have a wider variety of examples of higher type designs, but we suspect that they may be even more rare than $t$-designs.

Motivated by the fact that every $t$-design is automatically a $t-1$-design [12, Thm. 2.9], we ask when a $\tau$-design is also a $\mu$-design, $\mu \unrhd \tau$. This leads to the study of the basic relations and structure constants of $\operatorname{Hom}_{\mathbb{Z} S_{n}}(\mathbb{Z} B, \mathbb{Z} B)$ viewed as a combinatorial object. This study is certainly not as straightforward as in case $m=2$. For example, the incidence maps and projections defined in section 2 do not span an algebra. Indeed, the intervals $[1122,1233]$ and $[1122,3123]$ contain a different number of sequences of type $\left(2,1^{2}\right)$. In order salvage something, we say

Definition 5.2 A triple $(\lambda, \mu, \nu)$ of partitions of $n$ is balanced if there is an integer $a_{\lambda, \mu, \nu} \neq$ 0 so that

$$
\pi_{\nu} \psi^{\mu} \pi_{\mu} \psi^{\lambda}=a_{\lambda, \mu, \nu} \pi_{\nu} \psi^{\lambda} \text { or equivalently } \pi_{\lambda} \phi^{\mu} \pi_{\mu} \psi^{\nu}=a_{\lambda, \mu, \nu} \pi_{\lambda} \phi^{\nu} .
$$

Thus $a_{\lambda, \mu, \nu}$ is the number of $\mathbf{m} \in B^{\mu}$ such that $\mathbf{n} \geq \mathbf{m} \geq \mathbf{l}$ for arbitrary $\mathbf{n} \in B^{\nu}, 1 \in B^{\lambda}$ with $\mathbf{n} \geq 1$. The requirement $a_{\lambda, \mu, \nu} \neq 0$ implies that $\lambda \unrhd \mu \unrhd \nu$.

Given partitions $\lambda \unrhd \nu$ there are many (but as shown above, not all) intermediate partitions $\mu$ for which $(\lambda, \mu, \nu)$ is balanced. For instance, suppose $\lambda, \mu$ and $\nu$ are partitions of $n$ and $\lambda_{s}=\mu_{s}=\nu_{s}$ for all $s$ unless $s \in\{t-1, t\}$, and that $\lambda_{t-1} \geq \mu_{t-1} \geq \nu_{t-1}$. Then for $\mathbf{l} \in B^{\lambda}, \mathbf{m} \in B^{\mu}$ and $\mathbf{n} \in B^{\nu}$, the condition $\mathbf{l} \leq \mathbf{m} \leq \mathbf{n}$ implies that the associated flags $\mathbf{L}=\left\{L_{1} \supseteq \cdots \supseteq L_{m}\right\}, \mathbf{M}=\left\{M_{1} \supseteq \cdots \supseteq M_{m}\right\}$ and $\mathbf{N}=\left\{N_{1} \supseteq \cdots \supseteq N_{m}\right\}$ coincide 
except that $L_{t} \subseteq M_{t} \subseteq N_{t}$ (or equivalently that the sequences $1, \mathbf{m}$ and $\mathbf{n}$ coincide at all entries different from $t-1$ and $t)$. Ignore the entries not in $L_{t-1} \backslash L_{t+1}$ and reduce to the situation of 2-part partitions. By, for example Wilson [13, 3.1], $(\lambda, \mu, \nu)$ is balanced and $a_{\lambda, \mu, \nu}=\left(\begin{array}{c}\nu_{t}-\lambda_{t} \\ \mu_{t}-\lambda_{t}\end{array}\right)$. A natural extension of this example leads to the following

Definition 5.3 Let $\lambda$ and $\nu$ be partitions of $n$ with $\lambda \unrhd \nu$. We define the natural balanced sequence $\left(\lambda^{(t)}\right)$ from $\lambda$ to $\nu$ by

$$
\lambda_{s}^{(t)}:=\left\{\begin{array}{lr}
\nu_{s} & \text { if } s<t \\
n-\sum_{s>t} \lambda_{s}-\sum_{s<t} \nu_{s} & s=t \\
\lambda_{s} & s>t .
\end{array}\right.
$$

For the natural balanced sequence $\left(\lambda^{(t)}\right)$ from $\lambda$ to $\nu, \lambda=\lambda^{(1)}, \lambda^{(t)} \unrhd \lambda^{(t+1)}$ for all $t \geq 1$ and $\lambda^{(m)}=\nu$ if $\nu$ has $m$ parts. For instance, the natural balanced sequence from $(5,1)$ to $\left(2^{2}, 1^{2}\right)$ is $(5,1) \triangleright(2,4) \triangleright\left(2^{3}\right) \triangleright\left(2^{2}, 1^{2}\right)$.

Theorem 5.4 Let $\mu \unrhd \tau \unrhd \nu$ and let $\left(\mu=\mu^{(1)}, \cdots, \mu^{(r)}=\tau\right)$ be the natural balanced sequence from $\mu$ to $\tau$. Take $\mathbf{M} \leq \mathbf{T}$ for $\mathbf{M}$ of type $\mu$ and $\mathbf{T}$ of type $\tau$.

(1) There exist unique flags $\mathbf{M}=\mathbf{M}^{(1)} \leq \cdots \leq \mathbf{M}^{(r)}=\mathbf{T}$ with $\mathbf{M}^{(t)}$ of type $\mu^{(t)}$, so $\pi_{\tau} \phi^{\mu}=\pi_{\tau} \phi^{\mu^{(r-1)}} \pi_{\mu^{(r-1)}} \phi^{\mu^{(r-2)}} \cdots \pi_{\mu^{(2)}} \phi^{\mu}$ and $\pi_{\mu} \psi^{\tau}=\pi_{\mu} \psi^{\mu^{(2)}} \cdots \pi_{\mu^{(r-1)}} \psi^{\tau}$.

(2) Suppose $(\mu, \tau, \nu)$ is balanced and $S$ is a $\tau$-design of type $\nu$. Then $S$ is a $\mu$-design of type $\nu$ and $\lambda_{\mu}=\lambda_{\tau} a_{\mu, \tau}^{\Pi} / a_{\mu, \tau, \nu}$ is integral, where

$$
a_{\mu, \tau}^{\Pi}:=|\{\mathbf{S} \mid \mathbf{M} \leq \mathbf{S}, \underline{t y p}(\mathbf{S})=\tau\}|=\prod_{t=1}^{r-1}\left(\begin{array}{c}
\sum_{s=1}^{t}\left(\mu_{s}-\tau_{s}\right)+\tau_{t} \\
\tau_{t}
\end{array}\right) .
$$

Proof: There exists a flag $\mathbf{M}^{(t)}$ of type $\mu^{(t)}$ so that its first $t-1$ parts coincide with $\mathbf{T}$ and its parts $t+s$ coincide with $\mathbf{M}$ for all $s>0$. So $\mathbf{M} \leq \mathbf{M}^{(t)} \leq \mathbf{T}$ and $\mathbf{M}^{(t)}$ is uniquely determined with this property. Hence there is a unique chain $\mathbf{M}=\mathbf{M}^{(1)} \leq \cdots \leq \mathbf{M}^{(r)}=$ T with $\mathbf{M}^{(t)}$ of type $\mu^{(t)}(1 \leq t \leq r)$. Part one follows.

The map $\pi_{\mu} \psi^{\nu}: \mathbb{Z} B^{\nu} \rightarrow \mathbb{Z} B^{\mu}$ has a matrix with integer entries when expressed relative to the natural bases $B^{\nu}$ and $B^{\mu}$ and $S \in \mathbb{Z} B^{\nu}$ has integer coordinates. Therefore, $\pi_{\mu} \psi^{\nu}(S) \in \mathbb{Z} B^{\mu}$ has integer coordinates as well. Since $(\mu, \tau, \nu)$ is balanced, $a_{\mu, \tau, \nu} \pi_{\mu} \psi^{\nu}(S)=\pi_{\mu} \psi^{\tau} \pi_{\tau} \psi^{\nu}(S)=\mu_{\tau} \pi_{\mu} \psi^{\tau}\left(B^{\tau}\right)$. But for each flag $\mathbf{M}$ of type $\mu$, the number of flags $\mathbf{S}$ of type $\tau$ with $\mathbf{M} \leq \mathbf{S}$ is $a_{\mu, \tau}^{\Pi}$ and so $\lambda_{\tau} \pi_{\lambda} \psi^{\tau}\left(B^{\tau}\right)=\lambda_{\tau} a_{\mu, \tau}^{\Pi} B^{\mu}$.

It remains to compute $a_{\mu, \tau}^{n}$. To do this return to the notation of the first part and let $\mathbf{M}=\mathbf{M}^{(1)} \leq \cdots \leq \mathbf{M}^{(r)}=\mathbf{T}$ be the unique chain with $\mathbf{M}^{(t)}$ of type $\mu^{(t)}$ appearing in part one. Count the number of choices for $\mathbf{T}$ by counting the number of choices for $\mathbf{M}^{(t+1)}$, given $\mathbf{M}=\mathbf{M}^{(1)} \leq \cdots \leq \mathbf{M}^{(t)}$ and taking the product of these. Since $\mu^{(t)}$ and $\mu^{(t+1)}$ agree in all parts except the $t$-th and $(t+1)$-th part, there are exactly $\left(\begin{array}{c}\mu_{t}^{(t)} \\ \tau_{l}\end{array}\right)$ such choices. 
Theorem 5.5 Set $\mu=\left(\tau_{1}-1, \tau_{2}+1, \tau_{3}, \ldots, \tau_{m}\right)$. A $\tau$-design $S$ of type $\nu$ is uniquely reconstructible from $\left\{\pi_{\xi} \psi^{\nu}(S) \mid \xi \notin \mu\right\}$, if fewer than $\prod_{s \geq 1} s^{\tau_{*}}$ blocks are lost.

Proof: If $S$ and $S^{\prime}$ are two $\tau$-designs that agree in all of the indicated ways, then $S-S^{\prime} \in$ $\mathbb{Z} B^{\nu} \kappa_{\mu}$. The result follows from Theorem 4.6 and Theorem 2.4.4 as in Corollary 3.2.

The required list of " $\xi$-shadows" in Theorem 5.5 is much to large. At least the $\zeta$ for which there is a $\xi$ such that $(\zeta, \xi, \nu)$ is balanced are unnecessary. Exactly what a minimal sufficient list might be is a very interesting question.

\subsection{Codes}

As already noted at the beginning of Section 4 , because our codes are defined over $\mathbb{Z}$, it would be surprising if they had excellent error correction properties. If these codes are of practical value it must be because of a simple decoding procedure. None the less, they are closely related to the widely used Reed Muller codes and we take this opportunity to make this point.

Adopt the "algebraic mode" of presenting the natural permutation representations of $S_{n}$ from Section 2.1. Let $\mathbb{Z}\left[x_{1}, \ldots, x_{n}\right]^{<m}$ denote the $\mathbb{Z}$-polynomials (not polynomial functions) in $n$ variables $\left\{x_{i}\right\}$ of degree less than $m$ in each variable. Recall that the monomial $p(\mathbf{n}):=\prod x_{j}^{n_{j}-1} \in \mathbb{Z}\left[x_{1}, \ldots, x_{n}\right]^{<m}$ is associated with the sequence $\mathbf{n}=$ $\left(n_{1}, \ldots, n_{n}\right)$ and the associated tabloid has $s$-th part $\left\{j \mid n_{j}=s\right\}$. The type of $\mathbf{n}$ is $\nu$ where $\nu_{s}=\left|\left\{j \mid n_{j}=s\right\}\right|$.

The degree $\partial(\nu)=\sum(i-1) \nu_{i}$ of $p(\mathbf{n}), \mathbf{n} \in B^{\nu}$, depends only on $\nu$ (and equals the length of a covering chain from $(n)$ to $\nu$ in the domination poset of partitions of $n$ ). Unfortunately, the possible types $\nu$ for which $\partial(\nu)=k$ do not seem easy to describe in general and consequently clear connections with, for example, the polynomial codes of Kasami, Lin and Peterson [9] are awkward to establish. However, because the number of parts in a partition is bounded by $m$ in this discussion, we are able to make some connections.

The incidence map $\psi^{\nu}$ defined in Section 2.2 takes $\mathbf{n} \in B^{\nu}$ to the sum of all sequences that are termwise, less than or equal to $\mathbf{n}$ (or equivalently, all sequences where $j$ appears in a part no higher than $n_{j}$ ). These sequences are algebraically enumerated by the product:

(choices for 1st position) (choices for 2 nd position) $\cdots$ (choices for $n$-th position)

$$
\left(1+x_{1}+\cdots+x_{1}^{n_{1}}\right)\left(1+x_{2}+\cdots+x_{2}^{n_{2}}\right) \cdots\left(1+x_{n}+\cdots+x_{n}^{n_{n}}\right) .
$$

Since this formula makes no explicit mention of $\nu$, the $\mathbb{Z}$-homomorphism

$$
\begin{aligned}
\psi: \mathbb{Z}\left[x_{1}, \ldots, x_{n}\right]^{<m} \rightarrow \mathbb{Z}\left[x_{1}, \ldots, x_{n}\right]^{<m} & \\
\quad \text { defined by } \psi\left(\prod x_{j}^{n_{j}}\right) & =\prod \psi\left(x_{j}^{n_{j}}\right)=\prod \sum_{k=0}^{n_{j}} x^{k}
\end{aligned}
$$

is a global algebraic version of the incidence map $\psi$. The other incidence map $\phi$ is given by the $\mathbb{Z}$-homomorphism $\phi$ where $\phi\left(\prod x_{j}^{n_{j}}\right)=\prod \phi\left(x_{j}^{n_{j}}\right)=\prod x_{j}^{n_{j}} \psi\left(x_{j}^{m-1-n_{j}}\right)$.

Let $C_{m}$ denote the cyclic group of order $m$. Then $\mathbb{Z}\left[x_{1}, \ldots, x_{n}\right]<m$ may be identified with the group algebra $\mathbb{Z} C_{m}^{n}$. In case $m$ is prime, a $t$-flat $[1,13.7 .4]$ is a coset of a subgroup 
of $C_{m}^{n}$ having order $m^{t}$. It is helpful to extend this geometric language to the general case. For each subset $T \subseteq\{1, \ldots, n\}$ of cardinality $t$, define the associated coordinate $t$-space to be $\psi\left(\prod_{t \in T} x_{t}^{m-1}\right)=\phi\left(\prod_{s \notin T} x_{s}^{m-1}\right)$, and say a coordinate $t$-flat is anything of the form (monomial) $\cdots$ (coordinate $t$-space) $\in \mathbb{Z}\left[x_{1}, \ldots, x_{n}\right]^{<m}$.

Theorem 5.6 Let $E_{r}=\bigcap_{\nu \unrhd \omega(r)} \operatorname{ker} \pi_{\nu} \psi=\left\langle\text { im } \phi^{\nu} \mid \nu \unrhd \omega(r)\right\rangle^{\perp}$ where $\omega(r)=$ $\left(n-r 0^{m-2} r\right)$.

(1) Then $\nu \unrhd \omega(r)$ if and only if $\partial(\nu) \leq(m-1) r$.

(2) $E_{r}^{\perp}$ is generated by the characteristic functions of the coordinate $(n-r)$-flats.

Proof: By inspection, $\nu \unrhd \omega(r)$ if and only if $\nu_{1} \geq n-r$, and this is equivalent to $\partial(\nu) \leq \partial(\omega(r))$.

Suppose $S \cup T=\{1, \ldots, n\}$ and $|S|=n-r,|T|=r$. The monomial $\prod_{t \in T} x_{t}^{i_{t}}$ is in $\mathbb{Z}\left[x_{1}, \ldots, x_{n}\right]^{<m}$ if and only if $\underline{\operatorname{typ}}\left(\prod_{t \in T} x_{t}^{i_{t}}\right) \unrhd \omega(r)$ by part one. Part two follows from

$$
\prod_{t \in T} x_{t}^{i_{t}} \prod_{s \in S}\left(1+x_{s}+\cdots+x_{s}^{m-1}\right)=\phi\left(\prod_{t \in T, i_{t}=m} x_{t}^{m} \prod_{t \in T, i_{t}<m}\left(x_{t}^{i_{t}+1}-x_{t}^{i_{t}}\right)\right) .
$$

If $m$ is prime and $K=G F(m)$ be the Galois field with $m$ elements, then Theorem 5.6.2 implies that $E_{r} \otimes_{\mathbb{Z}} K$ contains the $m$-ary $(n-r-1)$-th order Euclidean geometry code over $K[1,13.7 .1]$. Of course the Euclidean Geometry codes are well studied in their own right and they admit a multistep majority decoding scheme that yields complete decoding in case $m=2$ [5]. It is quite instructive to compare this scheme with our poset approach. In case $m=2=|K|$, the code is the classical Reed Muller code and the two approaches coincide.

Corollary 5.7 Let $m=2$ and $K$ be an arbitrary field. Then the code $E_{r} \otimes_{\mathbb{Z}} K$ admits a complete $(n-r)$-step majority decoding scheme.

Proof: Since the partitions of $n$ into at most two parts form a totally ordered set and so $\nu \unrhd \omega(r)$ if and only if $\omega(r) \notin \nu$, so Theorem 2.4 .4 implies that $E_{r}=\mathbb{Z} B \kappa_{(n-r, r)}$. The global version of the parity checks defined in Lemma 2.10 have coordinates in $\{0,1\}$ and so are the same in all characteristics.

\section{References}

1. R.E. Blahut, Theory and Practice of Error Control Codes, Addison Wesley, Reading, Mass., 1983.

2. R.W. Carter and G. Lusztig, "On the Modular Representations of the General Linear and Symmetric Groups," Math Z. 136 (1974), 193-242.

3. C.W. Curtis and I. Reiner, Representation Theory of Finite Groups and Associative Algebras, Interscience, New York, 1962.

4. P. Frankl and J. Pach, "On the number of sets in a null $t$-design," European Journal of Combinatorics 4, (1983), 21-23. 
5. J.M. Goethals, "Threshold Decoding-A Tentative Survey," in: Coding and Complexity, ed. by G. Longo, Springer-Verlag, New York, 1975.

6. U. Eidt, "Über Moduln als Codes," Master Thesis, University of Bayreuth, Bayreuth, 1992.

7. G. James, "The Representation Theory of the Symmetric Groups," LNM 682, Springer-Verlag, New York, 1978.

8. G. James and A. Kerber, "The Representation Theory of the Symmetric Group," Encyclopedia of Mathematics and its Applications, Addison Wesley, London, 1981.

9. T. Kasami, S. Lin and W.W. Peterson, "Polynomial Codes," IEEE Trans. Info. Theory, 14 (1968), 808-814.

10. A. Kerber, "The Combinatorial Use of Finite Group Action," BI Wissenschaftsverlag, Manhiem 1991.

11. R.A. Liebler, "On Codes in the Natural Representation of the Symmetric Group," in: Combinatorics, Representations and Statistical Methods in Groups, Marcel Dekker, 1980.

12. F.J. Macwilliams and N.J.A. Sloane, The Theory of Error-Correcting Codes, North Holland, Amsterdam, 1978.

13. R.M. Wilson, "A Diagonal Form for the Incidence Matrices of $t$-Subsets vs. $k$-Subsets," Europ. $J$. Combinatorics 11, (1990), 609-615.

14. A. Wong, "Modules as Codes," Masters Thesis, Colorado State University, 1976. 